\title{
Analysis of variance tests for exponentiality of two distributions: Complete and censored samples
}

\author{
by
}

Hui Chen

A Thesis submitted to the Faculty of Graduate Studies of

The University of Manitoba

in partial fulfillment of the requirements for the degree of

MASTER OF SCIENCE

Department of Statistics

University of Manitoba

Winnipeg

Copyright (C) 2008 by Hui Chen 


\title{
THE UNIVERSITY OF MANITOBA \\ FACULTY OF GRADUATE STUDIES \\ $* * * * *$ \\ COPYRIGHT PERMISSION
}

\begin{abstract}
Analysis of variance tests for tests for exponentiality of two distributions: Complete and censored samples
\end{abstract}

BY

Hui Chen

A Thesis/Practicum submitted to the Faculty of Graduate Studies of The University of Manitoba in partial fulfillment of the requirement of the degree

Of

MASTER OF SCIENCE

Hui Chen $\mathbb{C} 2008$

Permission has been granted to the University of Manitoba Libraries to lend a copy of this thesis/practicum, to Library and Archives Canada (LAC) to lend a copy of this thesis/practicum, and to LAC's agent (UM/ProQuest) to microfilm, sell copies and to publish an abstract of this thesis/practicum.

This reproduction or copy of this thesis has been made available by authority of the copyright owner solely for the purpose of private study and research, and may only be reproduced and copied as permitted by copyright laws or with express written authorization from the copyright owner. 


\begin{abstract}
Using the principles of the $\mathrm{W}$-statistic for exponentiality of a single distribution (Shapiro and Wilk, 1972; Samanta and Schwarz, 1988) we develop procedures for testing a composite hypothesis of exponentiality of two distributions having the same scale parameter. The proposed V-exponential statistic for complete samples turns out to be a normalized ratio of the square of the generalized least squares estimator (also the minimum variance unbiased estimator) of the common scale parameter to a pooled sum of squares about the samples means. The V-exponential statistic is origin and scale invariant and has a null distribution that depends only on the sample sizes. We also prove some other important results relating to our proposed V-exponential statistic. Following the approach of Samanta and Schwarz (1988), the V-exponential statistic is then modified when one or both samples are censored. The modified test statistic has the same null distribution as in the uncensored case with a corresponding reduction in sample size(s).

Finally, following the approach of Stephens (1978), we propose a $V^{*}$-exponential statistic for testing exponentiality of two distributions for complete samples. In each case, we provide the empirical power results for various types of probability distributions considered under the alternative. We also compared the power results of the one-sample $W$-exponential test, two-sample $V$-exponential statistic and $V^{*}$ exponential statistic. We see that the obtained results are similar in each case. That is, the three tests seem comparable in terms of sensitivity.
\end{abstract}




\section{Acknowledgements}

I would like to express my deepest gratitude to my supervisors Dr. Saumen Mandal and Dr. Mrityunjay Samanta for their support, both academic and financial. I deeply appreciate their patience, stimulating suggestions and encouragements that gave me the possibility to complete this thesis.

I would like to thank my advisory committee members Dr. A. Leblanc and Dr. R. Thulasiram for their valuable feedback.

Special thanks go to all my friends who gave me support and encouragement.

Finally, I am deeply indebted to my parents for their support and love throughout this journey.

Hui Chen

The University of Manitoba

August, 2008 


\section{Table of Contents}

Abstract $\quad$ i

Acknowledgements $\quad$ ii

Table of Contents - iii.

List of Tables $\quad$ v

1 Introduction 1

2 One-sample W-statistics for Exponentiality 8

2.1 The W-exponential statistic for uncensored data . . . . . . . . . . 9

2.2 The W-exponential statistic for censored data . . . . . . . . . . 13

2.2.1 Origin unknown ................... 13

2.2 .2 Origin known . . . . . . . . . . . . . . 16

3 The Two-sample V-statistic for Exponentiality for Complete Sam$\begin{array}{ll}\text { ples } & 19\end{array}$

3.1 V-exponential statistic for complete samples . . . . . . . . . . . 19

3.2 Properties of the V-exponential statistic . . . . . . . . . . . . 24

3.3 Percentage Points of $V\left(n_{1}, n_{2}\right) \ldots \ldots \ldots \ldots$

3.4 Numerical Example . . . . . . . . . . . . . . . 43

4 Sensitivity Results for the Two-sample V-statistic 46 
5 The Two-sample V-statistic for Censored Samples . 49

6 Two-sample $V^{*}$-statistic for Exponentiality for Complete Samples 53

$6.1 V^{*}$-exponential statistic for complete sample . . . . . . . . . 53

6.2 Numerical Example . . . . . . . . . . . . . . . . . 56

6.3 Power Study . . . . . . . . . . . . . . . . . . 58

7 Conclusion $\quad 62$

$\begin{array}{ll}\text { Appendix } & 64\end{array}$

$\begin{array}{ll}\text { Bibliography } & 66\end{array}$ 


\section{List of Tables}

3.1 Percentage Points of V-Exponential . . . . . . . . . . . . . 30

4.1 Estimated Powers of the V-exponential test for selected sample sizes

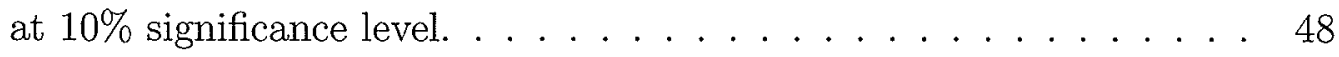

6.1 Estimated Powers of $V^{*}(11,10)$ and $W_{E}(20)$ at $5 \%$ significance level. . 60

6.2 Estimated Powers of $V$ and $V^{*}$ exponential tests for $n_{1}=15, n_{2}=15$, and $W$ test for $n=29$, at $10 \%$ significance level. . . . . . . . . 61 


\section{Chapter 1}

\section{Introduction}

When testing for goodness-of-fit, we take an observed sample and test how well it fits a given distribution. The general procedure consists in: (i) setting the null hypothesis, denoted by $H_{0}$, which states that a given random variable $X$ follows a probability distribution whose form is known and may depend on a number of known or unknown parameters, (ii) calculating a test statistic, which is some function of the data measuring the distance between the hypothesized distribution and the sample data, and (iii) making a decision about the acceptance or rejection of the null hypothesis.

There are many reasons to use goodness-of-fit tests. First, if the suggested probability model is correct, we have more confidence in our model for data generating process and on the parameters that describe the population. Secondly, the knowledge of the distribution for the data, allows us to use standard statistical testing and estimation

procedures, such as analysis of variance and the construction and calculation of confidence and prediction intervals. Finally, with the knowledge of the distribution, extreme tail probabilities can be computed.

There are several goodness-of-fit techniques. Graphical analysis is an informal procedure. One such technique is based on the probability plot, which is defined as the 
ratio of the number of observations less than or equal to $x_{i}$ (a realized value of the sample) to the total number of observations $n$. The probability distribution function is called empirical cumulative distribution function (i.e. ecdf). It is a simple tool which is easy to use on a piece of graph paper or using simple computer programs. It is less formal than the numerical techniques that will be presented later and usually is used to support the numerical testing procedures. It often gives a better understanding of the numerous relationships present in the data. The ecdf plot does not depend on any assumption about a hypothesized parametric distribution function. It has some advantages over the other statistical goodness-of-fit techniques since it gives immediate and direct information regarding the shape of the underlying distribution (i.e. skewness) and is an effective indicator of potential outliers. Also, most importantly, it can be used effectively for censored data. However, its sensitivity to random occurrences in the data sometimes leads to the wrong conclusion. We cannot solely rely on it, especially when the sample size is small.

The $\chi^{2}$ goodness-of-fit test is a classical statistical procedure. It was developed by Karl Pearson. It uses the comparison between the observed cell count and the corresponding expected value under the hypothesized distribution. The test statistic asymptotically follows a $\chi^{2}$ distribution with $c-k-1$ degrees of freedom. Here $c$ is the number of cells and $k$ is the number of estimated parameters for the distribution. The $\chi^{2}$ goodness-of-fit test is applied to binned data (i.e. data put into classes). Therefore, the value of the $\chi^{2}$ test statistic is sensitive to how the data is binned. Another disadvantage of the $\chi^{2}$ test is that it requires a sufficient sample size for the 
$\chi^{2}$ approximation to be valid. In practice, it is usually required that, the expected frequency should be at least 5 . Because of this, the test is not valid for small samples, and if some of the expected counts are less than five, one may need to combine some bins associated with the tails of the hypothesized distribution. These reductions discard some information, so that the $\chi^{2}$ goodness of fit test is less powerful than the other goodness-of-fit techniques. However, this test can be used for both continuous and discrete data, as well for the univariate and multivariate data. It is the most widely used goodness-of-fit test.

The Kolmogorov-Smirnov, Cramer-von Mises and Anderson-Darling tests are based on the distance between the empirical distribution function and the hypothesized distribution function. Let us take the Kolmogorov-Smirnov test as an example. From the empirical distribution function, we have the ratio of the number of observations less than or equal to $x_{i}$ to the total number of observations $n$ and $y_{i}$ are the corresponding ordered statistics. Hence the empirical distribution function for Kolmogorov-Smirnov test is a step function which will increase by $1 / n$ unit at the order of each data point. Kolmogorov-Smirnov test statistic is defined as the maximum distance between the empirical distribution function and the hypothesized population distribution function. The null distribution of the Kolmogorov-Smirnov test statistic does not depend on the underlying cumulative distribution function being tested. There is no limitation for the sample size, and it is an exact test. (i.e. not like $\chi^{2}$ goodness of fit test, which requires a sufficient sample size for the $\chi^{2}$ approximation to be valid). 
Goodness-of-fit tests for distributional assumptions have been a major study area for statistical research, especially for testing normality. Applications of the normal distribution can be mainly classified in two categories. First they relate to the class of statistics which are taken to be normally distributed due to the applicability of large sample theorems such as the Central Limit Theorem (Rao, 1973). Secondly, when the normal distribution is assumed, it can be applied to the appropriate mathematical model for the underlying phenomenon under investigation. The tests for normality can be classified into 5 groups: $\chi^{2}$ test, empirical distribution function tests, moment tests, regression tests, and miscellaneous tests. For example, as we mentioned before, the $\chi^{2}$ test uses the comparison between the observed cell counts and the corresponding expected values under the null hypothesis. For the normal distribution, the $\chi^{2}$ test statistic is calculated by grouping the hypothesized distribution (with known or estimated parameters) into a multinomial distribution of $\mathrm{M}$ cells, comparing the observed number of observations with the expected number of observations in each cell. The $\chi^{2}$ test is of historical interest and is continuously being modified.

The most commonly used goodness-of-fit test of normality is the Shapiro-Wilk test (1965) (so called W-statistic). The test considers a regression of the ordered sample observations on the expected values of the order statistics of a random sample from a standardized version of the hypothesized distribution. The W-test statistic for normality is defined by dividing the square of an appropriate linear combination of the sample order statistics (using the method of generalized least squares) by 
the usual symmetric estimate of variance. In fact the test considers the ratio of two estimates of the population variance and hence is also known as an analysis of variance test. This ratio is invariant for both location and scale. The exact distribution of $W$ statistic under the null hypothesis only depends on the sample size $n$, not on the location and scale parameters $\mu$ and $\sigma$. The exact distribution of the Wnormal statistic is unknown, but Shapiro and Wilk (1965) provided the percentage points for the test using Monte Carlo simulation. Using extensive Monte Carlo studies Shapiro and Wilk suggested that the critical region of the test is the lower tail area of the null distribution of $W$, that is, larger values of $W$ (i.e. values close to 1$)$ indicate normality. This $\mathrm{W}$-statistic is very simple to calculate when the table of appropriate linear coefficients is available. Even for small samples $(n<20)$, it is found that the test is quite sensitive (powerful) against a wide range of alternatives. Shapiro and Wilk (1965) provided the power results obtained from different goodness of fit tests. They concluded that the W-statistic is more powerful than other tests for skewed alternatives. Unfortunately, for large sample sizes, it may be difficult to determine the percentage points and may need a necessary value of the multiplier in the numerator for the test statistic.

From a general viewpoint, the procedure used to derive the W-statistic for normality can be applied to derive tests for other distributional assumptions, such as the exponential distribution. Using these principles, an analysis of variance test for exponentiality of a distribution based on a complete sample has been proposed by Shapiro and Wilk (1972). The W-exponential statistic is defined to be the ratio 
of the squared difference between the sample mean and the smallest observation to the usual symmetric sum of squares about the mean. Similar to the W-normal statistic, the $W$-exponential statistic is usable for testing various composite or simple hypotheses of exponentiality. The W-statistic for exponentiality leads to a two-tailed test. This is because the W-exponential statistic may take either low or high values depending on the properties of alternative distribution. Compared with other goodness-of-fit tests, the W-exponential statistic seems to be more powerful over a wide range of alternatives. A modified W-exponential statistic has been proposed by Samanta and Schwarz (1988). This modified statistic is applicable when the sample is censored. This will be discussed in detail in Chapter 2 of this thesis.

So far the test procedures available in the literature are all based on one single sample from one population. The proposed test statistic for testing the composite hypothesis of exponentiality of two distributions is developed in Chapter 3. We consider the null hypothesis that two independent random samples come from two exponential distributions with different unknown location parameters, but with the same unknown scale parameter, against the alternative hypothesis that the common form of these two distributions is not exponential. Using the principles used for the construction of the W-statistic for exponentiality, we propose a V-exponential statistic that turns out to be a normalized ratio of the square of the generalized least squares estimate of the common scale parameter based on the order statistics of independent random samples from the standard exponential distribution, to a pooled sum of squares about the sample means. This V-statistic is origin and scale 
invariant. The null distribution of the $\mathrm{V}$-exponential statistic is shown to depend only on the sample sizes. We also prove some other important results relating to our proposed V-exponential statistic. Tables of empirical percentage points of the V-statistic are constructed for various combinations of sample sizes by using Monte Carlo simulation. We give some numerical examples to illustrate the applications of the proposed test. Chapter 4 provides the empirical power results for various types of probability distributions under the alternative hypothesis. Chapter 5 deals with a modified test statistic using the approach of Samanta and Schwarz (1988) when one or both samples are censored. In Chapter 6, we use Stephens' (1978) approach and propose a second test statistic called the $V^{*}$-exponential statistic, that can be used in the same context. The null distribution of $V^{*}$-exponential statistic is the same as the W-exponential statistic of Shapiro and Wilk (1972) corresponding to an appropriately modified sample size. Numerical example and power studies of the $V^{*}$-exponential statistic are also included. We also compare the power results of the one-sample $W$-exponential test, two-sample $V$-exponential and $V^{*}$-exponential tests. We see that the results are close to each other, that is, the three tests ( $W$ exponential, $V$-exponential and $V^{*}$-exponential statistics) are comparable in terms of their sensitivity results. Concluding remarks are given in Chapter 7. 


\section{Chapter 2}

\section{One-sample W-statistics for Exponentiality}

There has been an extensive literature on goodness-of-fit tests for exponentiality. Important references include Shapiro and Wilk (1972), Anderson and Darling (1952), Bartholomew (1957), Cox and Lewis (1966), Darling (1953), Epstein (1960), Jackson (1967), and Stephens (1978). Recently, the exponentiality testing is most used for the time-constructed problem, such as waiting time. In this chapter, we discuss the one-sample test procedures for exponentiality due to Shapiro and Wilk (1972). The principle underlying the test procedures and the properties of the test statistic are similar to the W-statistic for normality (Shapiro and Wilk, 1965, 1968).

We have the general exponential distribution which has the density function defined as follows:

$$
f(x)= \begin{cases}\beta^{-1} \exp \{-(x-\alpha) / \beta\}, & x \geq \alpha, \\ 0, & \text { otherwise. }\end{cases}
$$

where $\alpha$ is the location parameter $(-\infty<\alpha<\infty)$ and $\beta$ is the scale parameter $(\beta>0)$.

Note that a random variable $X$ having density function $f$ is such that

1. $\mu_{x}=E(X)=\alpha+\beta$,

2. $\sigma_{x}^{2}=\operatorname{Var}(X)=\beta^{2}$, 
3. when $\alpha=0$ and $\beta=1, X$ admits the standard exponential distribution,

$$
f(x)= \begin{cases}e^{-x}, & x \geq 0 \\ 0, & \text { otherwise }\end{cases}
$$

\subsection{The W-exponential statistic for uncensored data}

Suppose we define $X_{1} \leq X_{2} \leq \ldots \leq X_{n}$ to be the order statistics of a sample of size $n$ obtained from a standard exponential distribution. Let the expected value of $X_{i}$ be $m_{i}$, that is,

$$
E\left(X_{i}\right)=m_{i}, \quad i=1,2, \cdots, n
$$

We have the covariance between $X_{i}$ and $X_{j}$ as

$$
\operatorname{Cov}\left(X_{i}, X_{j}\right)=v_{i j}=E\left[\left(X_{i}-m_{i}\right)\left(X_{j}-m_{j}\right)\right], \quad i, j=1,2, \cdots, n,
$$

and we write the expected values and covariances into vector and matrix form as follows

$$
\begin{gathered}
m^{\prime}=\left(m_{1}, m_{2}, \cdots, m_{n}\right), \quad \text { where } m^{\prime} \text { is the transpose of the vector } m, \\
V=\left(v_{i j}\right), \quad \text { where } V \text { is a } n \times n \text { matrix. }
\end{gathered}
$$

Further suppose $Y_{1} \leq Y_{2} \leq \ldots \leq Y_{n}$ are the corresponding ordered observations from an exponential distribution with location parameter $\alpha$ and scale parameter $\beta$. Then we can write $Y_{i}$ in terms of $X_{i}$ as

$$
Y_{i}=\alpha+\beta X_{i}, \quad i=1,2, \cdots, n,
$$


where $X_{i}$ is from the standard exponential distribution with density function as given in (2.2). We also write $Y$ in vector form for $y$ as

$$
Y=\left(\begin{array}{c}
Y_{1} \\
Y_{2} \\
\vdots \\
Y_{n}
\end{array}\right) .
$$

Now, we apply the generalized least squares theory (Aitken, 1935, Lloyd, 1952) to find the least square estimators of $\alpha$ and $\beta$. We have

$$
\left(\begin{array}{c}
\widehat{\alpha} \\
\widehat{\beta}
\end{array}\right)=\left\{(1 \mid m)^{\prime} V^{-1}(1 \mid m)\right\}^{-1}(1 \mid m)^{\prime} V^{-1} Y,
$$

since

$$
\begin{aligned}
\left\{(1 \mid m)^{\prime} V^{-1}(1 \mid m)\right\}^{-1} & =\left(\begin{array}{cc}
1^{\prime} V^{-1} 1 & 1^{\prime} V^{-1} m \\
1^{\prime} V^{-1} m & m^{\prime} V^{-1} m
\end{array}\right)^{-1} \\
& =\frac{1}{\operatorname{det}}\left(\begin{array}{cc}
m^{\prime} V^{-1} m & -1^{\prime} V^{-1} m \\
-1^{\prime} V^{-1} m & 1^{\prime} V^{-1} 1
\end{array}\right),
\end{aligned}
$$

where

$$
\begin{gathered}
\operatorname{det}=\left\{\left(1^{\prime} V^{-1} 1\right)\left(m^{\prime} V^{-1} m\right)-\left(1^{\prime} V^{-1} m\right)^{2}\right\}, \\
1^{\prime}=(1,1, \cdots, 1) .
\end{gathered}
$$

Hence, we have

$$
\widehat{\beta}=\frac{1^{\prime} V^{-1}\left(1 m^{\prime}-m 1^{\prime}\right) V^{-1} Y}{\left\{\left(1^{\prime} V^{-1} 1\right)\left(m^{\prime} V^{-1} m\right)-\left(1^{\prime} V^{-1} m\right)^{2}\right\}} .
$$


From known properties of the exponential distribution (Kendall and Stuart, 1961), we have the following properties for $m_{i}$ and $\operatorname{Cov}\left(X_{i}, X_{j}\right)$

$$
\begin{aligned}
m_{i} & =\sum_{k=1}^{i}(n-k+1)^{-1}, \quad i=1,2, \cdots, n, \\
\operatorname{Cov}\left(X_{i}, X_{j}\right) & =\sum_{k=1}^{i}(n-k+1)^{-2}, \quad i \leq j, \\
\operatorname{Cov}\left(X_{i}, X_{j}\right) & =\sum_{k=1}^{j}(n-k+1)^{-2}, \quad i>j .
\end{aligned}
$$

After some algebraic work, we have some important Lemmas (Shapiro and Wilk, 1972) as following:

1. $m_{j}=\sum_{i=1}^{n} v_{i j}$, that is, $m^{\prime}=1^{\prime} V$

2. $\sum_{i=1}^{n} m_{i}=n$, that is, $1^{\prime} m=n$,

3. $1^{\prime} V^{-1}=\left(n^{2}, 0,0, \cdots, 0\right)$,

and Corollaries:

1. $1^{\prime}=m^{\prime} V^{-1}$,

2. $m^{\prime} V^{-1} m=n$,

3. $1^{\prime} V^{-1} m=n$,

4. $1^{\prime} V^{-1} 1=n^{2}$,

5. $1^{\prime} V^{-1} Y=n^{2} Y_{1}$ 
6. $\widehat{\beta}=n\left(\bar{Y}-Y_{1}\right) /(n-1)$, where $\bar{Y}=\sum_{i=1}^{n} Y_{i} / n$.

We obtain the W-exponential statistic by standardizing the squared $\widehat{\beta}$ with $S^{2}$, so the $\mathrm{W}$-statistic for testing the composite hypothesis for exponentiality is

$$
W_{E}=\frac{n\left(\bar{Y}-Y_{1}\right)^{2}}{(n-1) S^{2}}
$$

for the null hypothesis:

$$
H_{10}: F(y)=1-\exp \{-(y-\alpha) / \beta\}, \quad y \geq \alpha
$$

where

$$
S^{2}=\sum_{i=1}^{n}\left(Y_{i}-\bar{Y}\right)^{2}
$$

Note that

- $\mathrm{W}$ is used as a two-tailed statistic. It is invariant for both origin and scale parameters and hence, can be used for testing the composite hypothesis of exponentiality.

- From the mathematical point of view, the W-exponential statistic is bounded, with a maximum value of 1 and minimum value of $(n-1)^{-2}$ (see Shapiro, Wilk and Chen, 1968).

- From the statistical point of view, we know that $Y_{1}$ is a sufficient statistic for the origin parameter $\alpha$ and $\bar{Y}$ is the sufficient for the scale parameter $\beta$ 
(Lehmann, 1959). As we mentioned before, the W-statistic is invariant for both origin and scale. As a result, under the null hypothesis, we have that the W-exponential statistic is independent of $Y_{1}$ and $\bar{Y}$ (Basu, 1955). It depends only on the sample size $n$ (Hogg and Craig, 1956).

\subsection{The W-exponential statistic for censored data}

As previously mentioned the $\mathrm{W}$-exponential statistic is given by

$$
W_{E}=\frac{n\left(\bar{Y}-Y_{1}\right)^{2}}{(n-1) S^{2}}
$$

for the complete sample of size $n$ (i.e. uncensored sample).

Now consider the case of a censored sample. That is, in a random sample of size $n$, the $r_{1}$ smallest and $r_{2}$ largest observations are censored. Then there will be $n-r_{1}-r_{2}$ observations available. Samanta and Schwarz (1988) modified the W-exponential statistic according to two different situations, (i.e. origin unknown and known).

\subsubsection{Origin unknown}

We denote the normalized waiting times as

$$
T_{i}=(n-i+1)\left(Y_{i}-Y_{i-1}\right), \quad i=2,3, \cdots, n .
$$


We know that when $H_{10}$ is true, $T_{2}, T_{3}, \cdots, T_{n}$, are independent and identically distributed random variables from the exponential distribution function

$$
F(t)= \begin{cases}1-e^{-t / \beta}, & t \geq 0 \\ 0, & \text { otherwise }\end{cases}
$$

Now, let

$$
\begin{aligned}
U_{i} & =\frac{i Y_{i+1}-\left(Y_{1}+Y_{2}+\cdots+Y_{i}\right)}{\sqrt{i(i+1)}}, \quad i=1,2, \cdots, n-1, \\
U_{n} & =\frac{Y_{1}+Y_{2}+\cdots+Y_{n}}{\sqrt{n}}=\sqrt{n Y}
\end{aligned}
$$

which actually define an orthogonal transformation. If we write $U_{i}$ in terms of $T_{i}$, we get

$$
U_{i}=\frac{\sum_{j=1}^{i} \frac{j T_{j+1}}{n-j}}{\sqrt{i(i+1)}}, \quad i=1,2, \cdots, n-1
$$

Let

$$
a_{i j}^{(n)}=\frac{(j-1)}{(n-j+1)}, \quad(n \geq i \geq j \geq 2)
$$

with

$$
a_{i j}^{(n)}=a_{j i}^{(n)} \quad \text { for } i, j=2,3, \cdots, n
$$

Using the newly defined variables, the original W-exponential statistic from the uncensored data (we denote $W_{E}(n)$ to indicate it is from a complete sample of size 
n) can be written as:

$$
\begin{aligned}
W_{E}(n) & =\frac{\left(\sum_{i=2}^{n} T_{i}\right)^{2}}{n(n-1) \sum_{i=1}^{n-1} U_{i}^{2}} \\
= & \frac{\left(\sum_{i=2}^{n} T_{i}\right)^{2}}{(n-1) \sum_{i=2}^{n} \sum_{j=2}^{n} a_{i j}^{(n)} T_{i} T_{j}}
\end{aligned}
$$

Now consider the censored situation as we mentioned before, that is when $r_{1}$ smallest and $r_{2}$ largest observations are censored. In other words we have an effective sample of size $n-r_{1}-r_{2}$. That is $Y_{r_{1}+1} \leq Y_{r_{1}+2} \leq \ldots \leq Y_{n-r_{2}}$ available observations for testing the null hypothesis $H_{10}$ with the origin unknown. Then, the modified test statistic (denoted as $W_{1}$ ) is given by

$$
W_{1}=\frac{\left(\sum_{i=2}^{n-r_{1}-r_{2}} T_{r_{1}+i}\right)^{2}}{\left(n-r_{1}-r_{2}-1\right) \sum_{i=2}^{n-r_{1}-r_{2}} \sum_{j=2}^{n-r_{1}-r_{2}} a_{i j}^{\left(n-r_{1}-r_{2}\right)} T_{r_{1}+i} T_{r_{1}+j}}
$$

Note that

1. if there are no censored data, $W_{1}$ is equal to the original $W_{E}(n)$.

2. the distribution of $W_{1}$ is the same as that of $W_{E}\left(n-r_{1}-r_{2}\right)$ under the null hypothesis. 


\subsubsection{Origin known}

Let us consider the situation where the origin is known. Stephens (1978) extended the Shapiro and Wilk exponentiality test for testing the null hypothesis

$$
H_{20}: F(y)=\left\{\begin{array}{ll}
1-\exp \left\{-\left(y-\alpha_{0}\right) / \beta\right\}, & y \geq \alpha_{0}, \\
0, & \text { otherwise }
\end{array} \text { with } \alpha_{0}\right. \text { known }
$$

The extended W-exponential statistic is $W_{E}^{*}(n)$

$$
W_{E}^{*}(n)=\frac{\left\{\sum_{i=1}^{n}\left(Y_{i}-\alpha_{0}\right)\right\}^{2}}{n\left\{(n+1) \sum_{i=1}^{n}\left(Y_{i}-\alpha_{0}\right)^{2}-\left[\sum_{i=1}^{n}\left(Y_{i}-\alpha_{0}\right)\right]^{2}\right\}}
$$

Let

$$
Z_{i}=Y_{i}-\alpha_{0}, \quad i=1,2, \cdots, n
$$

and the normalized waiting times $T_{i}$ for $i=2,3, \cdots, n$, as

$$
T_{i}=(n-i+1)\left(Y_{i}-Y_{i-1}\right)=(n-i+1)\left(Z_{i}-Z_{i-1}\right)
$$

and define $T_{1}$ as

$$
T_{1}=n Z_{1}=n\left(Y_{1}-\alpha_{0}\right)
$$

We also know, when $H_{20}$ is true, $T_{1}, T_{2}, \cdots, T_{n}$ are $i i d$ random variables following the exponential distribution function

$$
F(t)= \begin{cases}1-e^{-t / \beta}, & t \geq 0 \\ 0, & \text { otherwise }\end{cases}
$$


If we write $W_{E}^{*}(n)$ in terms of $T_{i}$ and $a_{i j}$, we get

$$
\begin{aligned}
W_{E}^{*}(n) & =\frac{\left(\sum_{i=1}^{n} T_{i}\right)^{2}}{(n+1) \sum_{i=2}^{n} \sum_{j=2}^{n} a_{i j}^{(n)} T_{i} T_{j}+\left(\sum_{i=1}^{n} T_{i}\right)^{2}} \\
= & \frac{\left(\sum_{i=2}^{n+1} T_{i-1}\right)^{2}}{n \sum_{i=2}^{n+1} \sum_{j=2}^{n+1} a_{i j}^{(n+1)} T_{i-1} T_{j-1}}
\end{aligned}
$$

We can use this modified W-exponential statistic for the case where the origin $\alpha_{0}$ is known in the context of censored data. Suppose that, in the sample of size $n$; the $r_{2}$ largest observations are censored. Then, we have an effective sample of $n-r_{2}$ available observations, that is $Y_{1} \leq Y_{2} \leq \ldots \leq Y_{n-r_{2}}$ for testing the null hypothesis $H_{20}$ with the origin known. The modified test statistic (denotes as $W_{2}$ ) can be written as:

$$
W_{2}=\frac{\left(\sum_{i=2}^{n-r_{2}+1} T_{i-1}\right)^{2}}{\left(n-r_{2}\right) \sum_{i=2}^{n-r_{2}+1} \sum_{j=2}^{n-r_{2}+1} a_{i j}^{\left(n-r_{2}+1\right)} T_{i-1} T_{j-1}}
$$

Note that

1. if there are no censored data, $W_{2}$ is equal to the original $W_{E}^{*}(n)$. 
2. following Stephens (1978), the distribution of $W_{2}$ is the same as that of $W_{E}^{*}\left(n-r_{2}+1\right)$ under the null hypothesis. 


\section{Chapter 3}

\section{The Two-sample V-statistic for Exponentiality for Complete Samples}

Based on the principles used by Shapiro and Wilk(1972) to derive W-statistic for testing exponentiality and by Samanta and Schwarz(1988) to derive the modified W-exponential statistic for censored data, we develop the V-exponential statistic for testing exponentiality of two distributions.

\subsection{V-exponential statistic for complete samples}

Suppose for $i=1,2, Y_{i 1} \leq Y_{i 2} \leq \ldots \leq Y_{i n_{i}}$ are the order statistics of a random sample from a population with distribution function $F_{i}(y)$. In this chapter we wish to test the null hypothesis:

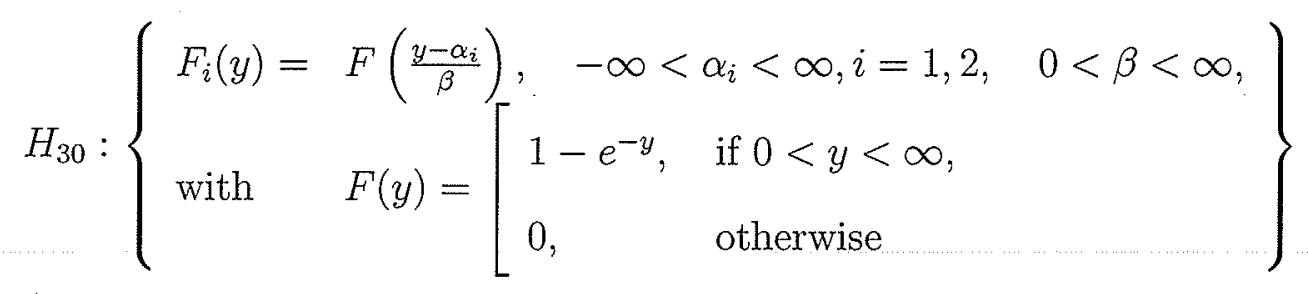

against the alternative hypothesis 
Chapter 3. The Two-sample V-statistic for Exponentiality for Complete Samples 20

$$
H_{3 a}:\left\{\begin{array}{ll}
F_{i}(y)= & F\left(\frac{y-\alpha_{i}}{\beta}\right) \\
\text { with } \quad F(y) \neq 1-e^{-y}, \quad \text { for some } y
\end{array}\right\}
$$

It is known that if the null hypothesis $H_{30}$ is true, then a test of the different null hypothesis

$$
H_{40}: \alpha_{1}=\alpha_{2}
$$

can be done by using an F-test.

Let us write

$$
Y_{i j}=\alpha_{i}+\beta X_{i j}, \quad \text { with } i=1,2 \quad j=1,2, \cdots, n_{i},
$$

where for each $i=1,2, \quad X_{i 1} \leq X_{i 2} \leq \ldots \leq X_{i n_{i}}$ are the order statistics of a random sample of size $n_{i}$ from a standard exponential distribution $F(x)$ as defined above (or in 2.2)

Note that $x_{i j}$ and $x_{l k}$ are independent when $i \neq l$.

Under $H_{30}$, it follows that for each $i=1,2, \quad Y_{i 1} \leq Y_{i 2} \leq \ldots \leq Y_{i n_{i}}$ are the corresponding order statistics of a random sample of size $n_{i}$ obtained from an exponential distribution with location parameter $\alpha_{i}$ and scale parameter $\beta$. 
Chapter 3. The Two-sample V-statistic for Exponentiality for Complete Samples 21

We define the two vectors $Y_{1}$ and $Y_{2}$ as

$$
Y_{1}=\left(\begin{array}{c}
Y_{11} \\
Y_{12} \\
\vdots \\
Y_{1 n_{1}}
\end{array}\right), \quad Y_{2}=\left(\begin{array}{c}
Y_{21} \\
Y_{22} \\
\vdots \\
Y_{2 n_{2}}
\end{array}\right)
$$

and we write the general $Y$ vector as

$$
Y=\left(\begin{array}{c}
Y_{1} \\
Y_{2}
\end{array}\right) .
$$

As before we denote the expected value of $X_{i j}$ as $m_{i j}$, that is,

$$
E\left(X_{i j}\right)=m_{i j}, \quad i=1,2, \quad j=1,2, \cdots, n_{i}
$$

and the covariance between $X_{i j}$ and $X_{l k}$ as

$$
\operatorname{Cov}\left(X_{i j}, X_{l k}\right)= \begin{cases}0, & \text { for } i \neq l \\ w_{1 j k}, & \text { for } i=l=1, j, k=1,2, \cdots, n_{1} \\ w_{2 j k}, & \text { for } i=l=2, j, k=1,2, \cdots, n_{2}\end{cases}
$$

So for each sample we have the covariance matrix

$$
\begin{aligned}
& \operatorname{Cov}_{1}=\left(w_{1 j k}\right)_{n_{1} \times n_{1}} \\
& \operatorname{Cov}_{2}=\left(w_{2 j k}\right)_{n_{2} \times n_{2}} .
\end{aligned}
$$


Chapter 3. The Two-sample V-statistic for Exponentiality for Complete Samples 22

For convenience, we write the covariance matrix of $Y$ as

$$
Q=\left(\begin{array}{cc}
\operatorname{Cov}_{1} & 0 \\
0 & \operatorname{Cov}_{2}
\end{array}\right)
$$

If we take the expectation of both sides of

$$
Y_{i j}=\alpha_{i}+\beta X_{i j},
$$

we will have, in matrix form,

$$
E(Y)=p \theta,
$$

where

$$
p=\left(\begin{array}{ccc}
1 & 0 & m_{11} \\
1 & 0 & m_{12} \\
\vdots & \vdots & \vdots \\
1 & 0 & m_{1 n_{1}} \\
0 & 1 & m_{21} \\
0 & 1 & m_{22} \\
\vdots & \vdots & \vdots \\
0 & 1 & m_{2 n_{2}}
\end{array}\right),
$$

and

$$
\theta=\left(\begin{array}{c}
\alpha_{1} \\
\alpha_{2} \\
\beta
\end{array}\right) .
$$

Now we apply the generalized least squares theory (Aitken, 1935, Lloyd, 1952) to 
Chapter 3. The Two-sample V-statistic for Exponentiality for Complete Samples 23

obtain the least squares estimators for $\alpha_{1}, \alpha_{2}$ and $\beta$. We have

$$
\widehat{\theta}=\left(\begin{array}{c}
\widehat{\alpha}_{1} \\
\widehat{\alpha}_{2} \\
\widehat{\beta}
\end{array}\right)=\left(p^{\prime} Q^{-1} p\right)^{-1} p^{\prime} Q^{-1} Y
$$

where $p^{\prime}$ is the transpose of $p$ matrix.

Using Lemmas 1 to 3 and Corollaries 1 to 5 , it can be shown that

$$
p^{\prime} Q^{-1} p=\left(\begin{array}{ccc}
n_{1}^{2} & 0 & n_{1} \\
0 & n_{2}^{2} & n_{2} \\
n_{1} & n_{2} & n_{1}+n_{2}
\end{array}\right)
$$

and

$$
p^{\prime} Q^{-1} Y=\left(\begin{array}{c}
n_{1}^{2} Y_{11} \\
n_{2}^{2} Y_{21} \\
\sum_{i=1}^{2} \sum_{j=1}^{n_{i}} Y_{i j}
\end{array}\right)
$$

Hence, we have the estimate $\widehat{\beta}$ for $\beta$, which is the product of the last row of $\left(p^{\prime} Q^{-1} p\right)^{-1}$ with the column vector $p^{\prime} Q^{-1} Y$, i.e.

$$
\widehat{\beta}=\frac{n_{1}\left(\bar{Y}_{1}-Y_{11}\right)+n_{2}\left(\bar{Y}_{2}-Y_{21}\right)}{\left(n_{1}+n_{2}-2\right)}
$$

where

$$
\bar{Y}_{i}=\sum_{j=1}^{n_{i}} Y_{i j} / n_{i}, \quad i=1,2 .
$$


Chapter 3. The Two-sample V-statistic for Exponentiality for Complete Samples 24

Note that $\widehat{\beta}$ is the minimum variance unbiased estimator (i.e. MVUE) for $\beta$ (Epstein and Sobel, 1954)

We obtain the V-exponential statistic as a normalized ratio of the squared $\widehat{\beta}$ to $S^{2}$. Hence, the resulting statistic for testing the composite hypothesis of exponentiality, given in (3.1) is

$$
V\left(n_{1}, n_{2}\right)=\frac{\left\{n_{1}\left(\bar{Y}_{1}-Y_{11}\right)+n_{2}\left(\bar{Y}_{2}-Y_{21}\right)\right\}^{2}}{2 n^{*}\left(n^{*}-1\right) S^{2}}
$$

where

$$
\begin{aligned}
S_{i}^{2} & =\sum_{j=1}^{n_{i}}\left(Y_{i j}-\bar{Y}_{i}\right)^{2}, \quad i=1,2, \\
S^{2} & =S_{1}^{2}+S_{2}^{2}, \\
n^{*} & =\max \left(n_{1}, n_{2}\right) .
\end{aligned}
$$

Note that, as an omnibus procedure, $V\left(n_{1}, n_{2}\right)$ is to be used as a two-tailed statistic.

\subsection{Properties of the $\mathrm{V}$-exponential statistic}

Based on the above two-sample V-statistic, we obtain the following important results.

THEOREM 1 .

$$
P\left\{1 / 2\left(n^{*}-1\right)^{2}<V\left(n_{1}, n_{2}\right) \leq 1\right\}=1
$$


Chapter 3. The Two-sample V-statistic for Exponentiality for Complete Samples 25

Proof.

According to Lemmas 5 and 6 in Shapiro and Wilk (1972), we note that for any two sample sequences

$$
\left\{n_{1}\left(\bar{Y}_{1}-Y_{11}\right)+n_{2}\left(\bar{Y}_{2}-Y_{21}\right)\right\}^{2} \leq 2 n^{*}\left(n^{*}-1\right) S^{2}
$$

and

$$
\begin{aligned}
\left\{n_{1}\left(\bar{Y}_{1}-Y_{11}\right)+n_{2}\left(\bar{Y}_{2}-Y_{21}\right)\right\}^{2} & \geq\left\{\frac{n_{1}}{\left(n_{1}-1\right)^{1 / 2}} S_{1}+\frac{n_{2}}{\left(n_{2}-1\right)^{1 / 2}} S_{2}\right\}^{2} \\
& >\frac{n^{*} S^{2}}{n^{*}-1}
\end{aligned}
$$

Therefore, we can write

$$
\begin{aligned}
\frac{\left\{n_{1}\left(\bar{Y}_{1}-Y_{11}\right)+n_{2}\left(\bar{Y}_{2}-Y_{21}\right)\right\}^{2}}{2 n^{*}\left(n^{*}-1\right) S^{2}} & >\frac{n^{*} S^{2}}{n^{*}-1} \cdot \frac{1}{2 n^{*}\left(n^{*}-1\right) S^{2}} \\
& >\frac{1}{2\left(n^{*}-1\right)^{2}}
\end{aligned}
$$

As Shapiro and Wilk (1965) proved, the W-exponential statistic is bounded with a maximum value of 1 and minimum value of $(n-1)^{-2}$. Hence, the $\mathrm{V}$-exponential statistic has the following similar property

$$
1 \geq \frac{\left\{n_{1}\left(\bar{Y}_{1}-Y_{11}\right)+n_{2}\left(\bar{Y}_{2}-Y_{21}\right)\right\}^{2}}{2 n^{*}\left(n^{*}-1\right) S^{2}}>\frac{1}{2\left(n^{*}-1\right)^{2}} .
$$

\section{THEOREM 2 .}

The null distribution of $V\left(n_{1}, n_{2}\right)$ and $V\left(n_{2}, n_{1}\right)$ are identical and depend only on $n_{1}$ and $n_{2}$, but not on $\alpha_{1}, \alpha_{2}$ and $\beta$. 
Chapter 3. The Two-sample V-statistic for Exponentiality for Complete Samples 26

Proof.

Following Samanta and Schwarz (1988) we define $T_{i j}$ as

$$
T_{i j}=\left(n_{i}-j+1\right)\left(Y_{i j}-Y_{i, j-1}\right), \quad j=2,3, \cdots, n_{i}, \quad i=1,2 .
$$

Under the null hypothesis $H_{30}, T_{i j} / \beta, \quad j=2,3, \cdots, n_{i}, \quad i=1,2$, are independent and identically distributed random variables with the standard exponential distribution.

Let

$$
\begin{aligned}
& a_{i j}^{\left(n_{1}\right)}=\frac{(j-1)}{\left(n_{1}-j+1\right)}, \quad\left(n_{1} \geq i \geq j \geq 2\right) \\
& a_{i j}^{\left(n_{1}\right)}=a_{j i}^{\left(n_{1}\right)} \text { for } i, j=2,3, \cdots, n_{1}, \\
& b_{i j}^{\left(n_{2}\right)}=\frac{(j-1)}{\left(n_{2}-j+1\right)}, \quad\left(n_{2} \geq i \geq j \geq 2\right) \\
& b_{i j}^{\left(n_{2}\right)}=b_{j i}^{\left(n_{2}\right)} \text { for } i, j=2,3, \cdots, n_{2},
\end{aligned}
$$

Then we can write the V-exponential statistic $V\left(n_{1}, n_{2}\right)$ in terms of the $T_{i j}$ 's as

$$
V\left(n_{1}, n_{2}\right)=\frac{\left\{\sum_{i=2}^{n_{1}} T_{1 i}+\sum_{i=2}^{n_{2}} T_{2 i}\right\}^{2}}{2 n^{*}\left(n^{*}-1\right)\left\{\left[\sum_{i=2}^{n_{1}} \sum_{j=2}^{n_{1}} a_{i j}^{\left(n_{1}\right)} T_{1 i} T_{1 j}\right] / n_{1}+\left[\sum_{i=2}^{n_{2}} \sum_{j=2}^{n_{2}} b_{i j}^{\left(n_{2}\right)} T_{2 i} T_{2 j}\right] / n_{2}\right\}}
$$

So we can see from the above representation that the distribution of $V\left(n_{1}, n_{2}\right)$ and $V\left(n_{2}, n_{1}\right)$ are identical and depend only on the sample sizes $n_{1}$ and $n_{2}$ but not on 
Chapter 3. The Two-sample V-statistic for Exponentiality for Complete Samples 27

the parameters $\alpha_{1}, \alpha_{2}$ and $\beta$. As a result, for the exponentially distributed samples, $\left(Y_{11}, Y_{21}, n_{1}\left(\bar{Y}_{1}-Y_{11}\right)+n_{2}\left(\bar{Y}_{2}-Y_{21}\right)\right)$ is a complete sufficient statistic for $\left(\alpha_{1}, \alpha_{2}\right.$,

$\beta$ ) (Basu, 1955). Hence the statistic $V\left(n_{1}, n_{2}\right)$ is statistically independent of $\left(Y_{11}\right.$, $\left.Y_{21}, n_{1}\left(\bar{Y}_{1}-Y_{11}\right)+n_{2}\left(\bar{Y}_{2}-Y_{21}\right)\right)$ under null hypothesis.

\section{THEOREM 3 .}

Under the null hypothesis $H_{30}$, the distribution function of $V(2,2)$ (i.e. when $n_{1}=2, n_{2}=2$ ) is given by

$$
H(v)=1-\left(v^{-1}-1\right)^{1 / 2}, \quad \frac{1}{2} \leq v \leq 1 .
$$

Proof.

We have

$$
V\left(n_{1}, n_{2}\right)=\frac{\left\{\sum_{i=2}^{n_{1}} T_{1 i}+\sum_{i=2}^{n_{2}} T_{2 i}\right\}^{2}}{2 n^{*}\left(n^{*}-1\right)\left\{\left[\sum_{i=2}^{n_{1}} \sum_{j=2}^{n_{1}} a_{i j}^{\left(n_{1}\right)} T_{1 i} T_{1 j}\right] / n_{1}+\left[\sum_{i=2}^{n_{2}} \sum_{j=2}^{n_{2}} b_{i j}^{\left(n_{2}\right)} T_{2 i} T_{2 j}\right] / n_{2}\right\}}
$$

When $n_{1}=2$ and $n_{2}=2$,

$$
V(2,2)=\frac{\left(S_{1}+S_{2}\right)^{2}}{2\left(S_{1}^{2}+S_{2}^{2}\right)},
$$

where

$$
\begin{aligned}
& S_{1}=\frac{T_{12}}{\beta}, \\
& S_{2}=\frac{T_{22}}{\beta} .
\end{aligned}
$$


Chapter 3. The Two-sample V-statistic for Exponentiality for Complete Samples 28

Note that $S_{1}$ and $S_{2}$ are independent and identically distributed random variables with the standard exponential distribution.

Now, suppose it is given that

$$
S_{1}+S_{2}=s
$$

in which case the conditional distribution of $S_{1}$ is uniform. Then, for $u$ satisfying

$$
\frac{s^{2}}{2} \leq u \leq s^{2}
$$

we have

$$
P\left(S_{1}^{2}+S_{2}^{2} \leq u \mid S_{1}+S_{2}=s\right)=\frac{\left\{2\left(u-s^{2} / 2\right)\right\}^{1 / 2}}{s} .
$$

Now, let

$$
r=\frac{u}{s^{2}}
$$

and note that for any $r$ satisfying $\frac{1}{2} \leq r \leq 1$, we have

$$
\begin{aligned}
P\left(\frac{S_{1}^{2}+S_{2}^{2}}{\left(S_{1}+S_{2}\right)^{2}} \leq r \mid S_{1}+S_{2}=s\right) & =P\left(S_{1}^{2}+S_{2}^{2} \leq r s^{2} \mid S_{1}+S_{2}=s\right) \\
& =(2 r-1)^{1 / 2}
\end{aligned}
$$

so that this probability does not depend on $s$.

We concluded that for any $v$ satisfying $\frac{1}{2} \leq v \leq 1$, the distribution function $H(v)$ of 
Chapter 3. The Two-sample V-statistic for Exponentiality for Complete Samples 29

$V(2,2)$ is given by

$$
\begin{aligned}
H(v) & =1-P(V(2,2) \geq v) \\
& =1-P\left(\frac{S_{1}^{2}+S_{2}^{2}}{\left(S_{1}+S_{2}\right)^{2}} \leq \frac{1}{2 v}\right) \\
& =1-\left(v^{-1}-1\right)^{1 / 2} .
\end{aligned}
$$

\subsection{Percentage Points of $V\left(n_{1}, n_{2}\right)$}

The distribution of $V\left(n_{1}, n_{2}\right)$ under the null hypothesis was studied by Monte Carlo simulation. We obtained the empirical cumulative distribution of $V\left(n_{1}, n_{2}\right)$ from 100000 random samples with sizes $\left(n_{1}, n_{2}\right)$ (i.e. $\left.n_{1}=2(1) 25, n_{2}=2(1) 25, n_{1} \leq n_{2}\right)$. Then we calculated the empirical percentage points of $V\left(n_{1}, n_{2}\right)$ from this empirical distribution. The random samples were simulated using the Package $R$. The $0.5,1$, $2.5,5,95,97.5,99,99.5$ empirical percentage points of $V\left(n_{1}, n_{2}\right)$ are given in Table 3.1 . 
Chapter 3. The Two-sample V-statistic for Exponentiality for Complete Samples 30

Table 3.1: Percentage Points of V-Exponential

\begin{tabular}{|cc|cccccccc|}
\hline$n_{1}$ & $n_{2}$ & 0.005 & 0.01 & 0.025 & 0.05 & 0.95 & 0.975 & 0.99 & 0.995 \\
\hline 2 & 2 & 0.5023 & 0.5047 & 0.5123 & 0.525 & 0.9975 & 0.9993 & 0.9998 & 0.99996 \\
2 & 3 & 0.1418 & 0.15 & 0.1666 & 0.1865 & 0.6205 & 0.6433 & 0.657 & 0.6617 \\
2 & 4 & 0.0752 & 0.0815 & 0.0949 & 0.1101 & 0.4575 & 0.499 & 0.5336 & 0.5511 \\
2 & 5 & 0.0513 & 0.0569 & 0.0672 & 0.0779 & 0.3399 & 0.3871 & 0.4378 & 0.4677 \\
2 & 6 & 0.038 & 0.0427 & 0.0515 & 0.0605 & 0.2663 & 0.3049 & 0.3509 & 0.3826 \\
2 & 7 & 0.0313 & 0.0354 & 0.0428 & 0.05 & 0.2138 & 0.2458 & 0.2873 & 0.3214 \\
2 & 8 & 0.0268 & 0.0302 & 0.0365 & 0.0427 & 0.1787 & 0.2035 & 0.2376 & 0.2661 \\
2 & 9 & 0.0231 & 0.0263 & 0.0314 & 0.0367 & 0.1514 & 0.1727 & 0.2003 & 0.2234 \\
2 & 10 & 0.0206 & 0.0233 & 0.0282 & 0.0331 & 0.1315 & 0.1488 & 0.1726 & 0.1902 \\
2 & 11 & 0.0188 & 0.0212 & 0.0256 & 0.0297 & 0.1141 & 0.1287 & 0.1487 & 0.1633 \\
2 & 12 & 0.0173 & 0.0195 & 0.0233 & 0.0269 & 0.1021 & 0.1156 & 0.1334 & 0.1469 \\
2 & 13 & 0.0161 & 0.0182 & 0.0216 & 0.0249 & 0.0912 & 0.1028 & 0.1184 & 0.1304 \\
2 & 14 & 0.0151 & 0.0168 & 0.0201 & 0.0232 & 0.0823 & 0.0927 & 0.1067 & 0.1167 \\
2 & 15 & 0.0141 & 0.0158 & 0.0186 & 0.0215 & 0.0761 & 0.0849 & 0.098 & 0.1067 \\
2 & 16 & 0.0132 & 0.0148 & 0.0176 & 0.0202 & 0.069 & 0.077 & 0.0876 & 0.0965 \\
2 & 17 & 0.0124 & 0.014 & 0.0165 & 0.0189 & 0.0634 & 0.071 & 0.081 & 0.0885 \\
2 & 18 & 0.0121 & 0.0136 & 0.0159 & 0.0181 & 0.0589 & 0.0651 & 0.074 & 0.0804 \\
2 & 19 & 0.0113 & 0.0126 & 0.0149 & 0.017 & 0.0549 & 0.0612 & 0.0692 & 0.075 \\
2 & 20 & 0.0108 & 0.0121 & 0.0142 & 0.0162 & 0.0513 & 0.0567 & 0.0638 & 0.0699 \\
2 & 21 & 0.0104 & 0.0117 & 0.0137 & 0.0155 & 0.048 & 0.0532 & 0.0601 & 0.0653 \\
2 & 22 & 0.0101 & 0.0112 & 0.0131 & 0.0148 & 0.0452 & 0.0497 & 0.0562 & 0.0609 \\
2 & 23 & 0.0097 & 0.0108 & 0.0125 & 0.0142 & 0.0426 & 0.0471 & 0.053 & 0.0573 \\
2 & 24 & 0.0093 & 0.0104 & 0.012 & 0.0136 & 0.0403 & 0.0445 & 0.0499 & 0.0537 \\
2 & 25 & 0.0088 & 0.0099 & 0.0115 & 0.0131 & 0.0383 & 0.0421 & 0.047 & 0.0511 \\
\hline
\end{tabular}


Chapter 3. The Two-sample V-statistic for Exponentiality for Complete Samples 31

\begin{tabular}{|cc|cccccccc|}
\hline$n_{1}$ & $n_{2}$ & 0.005 & 0.01 & 0.025 & 0.05 & 0.95 & 0.975 & 0.99 & 0.995 \\
\hline 3 & 3 & 0.1623 & 0.1739 & 0.1962 & 0.2221 & 0.8278 & 0.8857 & 0.9342 & 0.9562 \\
3 & 4 & 0.088 & 0.0969 & 0.1136 & 0.1307 & 0.531 & 0.5796 & 0.6329 & 0.6643 \\
3 & 5 & 0.0602 & 0.0676 & 0.0787 & 0.0917 & 0.3803 & 0.4271 & 0.481 & 0.5156 \\
3 & 6 & 0.0456 & 0.0508 & 0.0599 & 0.0698 & 0.2896 & 0.3254 & 0.3735 & 0.4067 \\
3 & 7 & 0.0366 & 0.0414 & 0.0493 & 0.0571 & 0.2309 & 0.2609 & 0.2991 & 0.3285 \\
3 & 8 & 0.0308 & 0.035 & 0.0418 & 0.0483 & 0.1909 & 0.2154 & 0.2506 & 0.2761 \\
3 & 9 & 0.0266 & 0.0297 & 0.0355 & 0.0412 & 0.1606 & 0.1818 & 0.2087 & 0.2312 \\
3 & 10 & 0.0239 & 0.0266 & 0.0316 & 0.0365 & 0.1383 & 0.1558 & 0.1794 & 0.1982 \\
3 & 11 & 0.0206 & 0.0236 & 0.0282 & 0.0325 & 0.1204 & 0.1355 & 0.1553 & 0.17 \\
3 & 12 & 0.0196 & 0.0219 & 0.0257 & 0.0297 & 0.108 & 0.1213 & 0.1395 & 0.1515 \\
3 & 13 & 0.0173 & 0.0196 & 0.0233 & 0.0269 & 0.0952 & 0.1068 & 0.1216 & 0.1339 \\
3 & 14 & 0.0165 & 0.0185 & 0.022 & 0.025 & 0.0863 & 0.0964 & 0.1102 & 0.1206 \\
3 & 15 & 0.0153 & 0.0173 & 0.0203 & 0.0232 & 0.0787 & 0.0874 & 0.0989 & 0.1079 \\
3 & 16 & 0.0141 & 0.016 & 0.0188 & 0.0216 & 0.0714 & 0.0793 & 0.0901 & 0.0983 \\
3 & 17 & 0.0135 & 0.0151 & 0.0178 & 0.0203 & 0.066 & 0.0735 & 0.0831 & 0.0901 \\
3 & 18 & 0.0127 & 0.0142 & 0.0167 & 0.0191 & 0.0611 & 0.0678 & 0.0769 & 0.0836 \\
3 & 19 & 0.012 & 0.0135 & 0.016 & 0.0181 & 0.0568 & 0.0627 & 0.0708 & 0.0765 \\
3 & 20 & 0.0117 & 0.0131 & 0.0152 & 0.0172 & 0.0529 & 0.0586 & 0.0662 & 0.0717 \\
3 & 21 & 0.011 & 0.0123 & 0.0144 & 0.0164 & 0.0499 & 0.0549 & 0.0617 & 0.0675 \\
3 & 22 & 0.0105 & 0.0118 & 0.0138 & 0.0156 & 0.0465 & 0.0512 & 0.0576 & 0.0626 \\
3 & 23 & 0.0102 & 0.0114 & 0.0133 & 0.015 & 0.0437 & 0.0481 & 0.0536 & 0.0575 \\
3 & 24 & 0.0098 & 0.0109 & 0.0127 & 0.0143 & 0.0415 & 0.0456 & 0.0509 & 0.0547 \\
3 & 25 & 0.0094 & 0.0104 & 0.0121 & 0.0137 & 0.0392 & 0.0429 & 0.0481 & 0.052 \\
\hline
\end{tabular}


Chapter 3. The Two-sample V-statistic for Exponentiality for Complete Samples 32

\begin{tabular}{|cc|cccccccc|}
\hline$n_{1}$ & $n_{2}$ & 0.005 & 0.01 & 0.025 & 0.05 & 0.95 & 0.975 & 0.99 & 0.995 \\
\hline 4 & 4 & 0.1019 & 0.1126 & 0.1326 & 0.1528 & 0.6233 & 0.6862 & 0.7645 & 0.8136 \\
4 & 5 & 0.0685 & 0.0759 & 0.0904 & 0.105 & 0.4322 & 0.4807 & 0.5421 & 0.5801 \\
4 & 6 & 0.0519 & 0.0583 & 0.0692 & 0.0798 & 0.3216 & 0.3608 & 0.4111 & 0.4467 \\
4 & 7 & 0.0416 & 0.0468 & 0.055 & 0.0638 & 0.251 & 0.2823 & 0.3236 & 0.3575 \\
4 & 8 & 0.0337 & 0.0384 & 0.0457 & 0.0529 & 0.2056 & 0.2305 & 0.2634 & 0.288 \\
4 & 9 & 0.0293 & 0.0332 & 0.0396 & 0.0455 & 0.1733 & 0.1952 & 0.2245 & 0.2441 \\
4 & 10 & 0.0259 & 0.0291 & 0.0345 & 0.0398 & 0.1479 & 0.1666 & 0.1907 & 0.2077 \\
4 & 11 & 0.0229 & 0.0258 & 0.0308 & 0.0355 & 0.1278 & 0.1437 & 0.1653 & 0.1798 \\
4 & 12 & 0.0211 & 0.0236 & 0.0279 & 0.0321 & 0.1129 & 0.1261 & 0.1437 & 0.1559 \\
4 & 13 & 0.0192 & 0.0215 & 0.0253 & 0.029 & 0.0997 & 0.1113 & 0.126 & 0.1363 \\
4 & 14 & 0.018 & 0.02 & 0.0235 & 0.0269 & 0.0905 & 0.1008 & 0.1143 & 0.1256 \\
4 & 15 & 0.0163 & 0.0183 & 0.0215 & 0.0247 & 0.082 & 0.0911 & 0.1028 & 0.1121 \\
4 & 16 & 0.015 & 0.0169 & 0.0199 & 0.0228 & 0.0748 & 0.0828 & 0.0934 & 0.1031 \\
4 & 17 & 0.0145 & 0.0163 & 0.0192 & 0.0217 & 0.0688 & 0.0761 & 0.0854 & 0.0932 \\
4 & 18 & 0.0135 & 0.0152 & 0.0177 & 0.0203 & 0.0631 & 0.0697 & 0.0789 & 0.085 \\
4 & 19 & 0.0131 & 0.0145 & 0.0169 & 0.0191 & 0.0585 & 0.0646 & 0.0721 & 0.0778 \\
4 & 20 & 0.0124 & 0.0137 & 0.016 & 0.0181 & 0.0548 & 0.0604 & 0.0677 & 0.0729 \\
4 & 21 & 0.0117 & 0.013 & 0.0152 & 0.0173 & 0.0509 & 0.0562 & 0.0628 & 0.0677 \\
4 & 22 & 0.0112 & 0.0125 & 0.0145 & 0.0164 & 0.0478 & 0.0524 & 0.0581 & 0.0626 \\
4 & 23 & 0.0107 & 0.012 & 0.014 & 0.0157 & 0.0453 & 0.0496 & 0.0551 & 0.0594 \\
4 & 24 & 0.0103 & 0.0114 & 0.0132 & 0.0149 & 0.0426 & 0.0467 & 0.0519 & 0.0559 \\
4 & 25 & 0.01 & 0.011 & 0.0127 & 0.0143 & 0.0401 & 0.0439 & 0.0487 & 0.0524 \\
5 & 5 & 0.079 & 0.0873 & 0.103 & 0.1197 & 0.4833 & 0.5399 & 0.6093 & 0.6572 \\
\hline
\end{tabular}


Chapter 3. The Two-sample V-statistic for Exponentiality for Complete Samples 33

\begin{tabular}{|cc|cccccccc|}
\hline$n_{1}$ & $n_{2}$ & 0.005 & 0.01 & 0.025 & 0.05 & 0.95 & 0.975 & 0.99 & 0.995 \\
\hline 5 & 6 & 0.0573 & 0.0647 & 0.0768 & 0.0894 & 0.3547 & 0.3974 & 0.4496 & 0.4904 \\
5 & 7 & 0.0464 & 0.0517 & 0.0616 & 0.0713 & 0.2739 & 0.3066 & 0.3524 & 0.3858 \\
5 & 8 & 0.0387 & 0.043 & 0.0508 & 0.0584 & 0.2213 & 0.248 & 0.2813 & 0.3072 \\
5 & 9 & 0.0327 & 0.0368 & 0.0434 & 0.0497 & 0.1833 & 0.2039 & 0.2312 & 0.2504 \\
5 & 10 & 0.0282 & 0.0319 & 0.0374 & 0.043 & 0.155 & 0.1738 & 0.1967 & 0.2132 \\
5 & 11 & 0.0248 & 0.028 & 0.0332 & 0.0383 & 0.1343 & 0.1495 & 0.1699 & 0.1876 \\
5 & 12 & 0.0227 & 0.0254 & 0.03 & 0.0346 & 0.1186 & 0.1325 & 0.1494 & 0.1643 \\
5 & 13 & 0.0206 & 0.0232 & 0.0275 & 0.0313 & 0.1048 & 0.1165 & 0.1327 & 0.1446 \\
5 & 14 & 0.0185 & 0.0209 & 0.0247 & 0.0284 & 0.0943 & 0.1044 & 0.1179 & 0.1278 \\
5 & 15 & 0.0177 & 0.0198 & 0.0231 & 0.0263 & 0.0848 & 0.094 & 0.1061 & 0.1156 \\
5 & 16 & 0.0163 & 0.0184 & 0.0214 & 0.0244 & 0.0772 & 0.0855 & 0.0954 & 0.1032 \\
5 & 17 & 0.0152 & 0.0169 & 0.0199 & 0.0227 & 0.071 & 0.0787 & 0.089 & 0.096 \\
5 & 18 & 0.0145 & 0.0161 & 0.0189 & 0.0214 & 0.0654 & 0.0721 & 0.081 & 0.0873 \\
5 & 19 & 0.0135 & 0.015 & 0.0177 & 0.0202 & 0.0607 & 0.0671 & 0.0747 & 0.0811 \\
5 & 20 & 0.013 & 0.0145 & 0.0167 & 0.0189 & 0.0569 & 0.0628 & 0.0698 & 0.0756 \\
5 & 21 & 0.0125 & 0.0138 & 0.016 & 0.0181 & 0.0528 & 0.0579 & 0.0645 & 0.0693 \\
5 & 22 & 0.0118 & 0.0131 & 0.0153 & 0.0171 & 0.0495 & 0.0547 & 0.0611 & 0.0664 \\
5 & 23 & 0.0114 & 0.0125 & 0.0145 & 0.0163 & 0.0465 & 0.051 & 0.0569 & 0.0612 \\
5 & 24 & 0.0107 & 0.012 & 0.0139 & 0.0157 & 0.0438 & 0.0478 & 0.053 & 0.0566 \\
5 & 25 & 0.0106 & 0.0116 & 0.0134 & 0.015 & 0.0414 & 0.0453 & 0.0503 & 0.0539 \\
6 & 6 & 0.0642 & 0.0729 & 0.0866 & 0.0996 & 0.3847 & 0.4329 & 0.4909 & 0.5323 \\
6 & 7 & 0.0519 & 0.0581 & 0.0679 & 0.0783 & 0.2939 & 0.3314 & 0.3748 & 0.4083 \\
6 & 8 & 0.0424 & 0.0467 & 0.0557 & 0.0643 & 0.2359 & 0.2642 & 0.2993 & 0.3249 \\
\hline & & & & & & & & & \\
5
\end{tabular}


Chapter 3. The Two-sample V-statistic for Exponentiality for Complete Samples 34

\begin{tabular}{|cc|cccccccc|}
\hline$n_{1}$ & $n_{2}$ & 0.005 & 0.01 & 0.025 & 0.05 & 0.95 & 0.975 & 0.99 & 0.995 \\
\hline 6 & 9 & 0.0351 & 0.0396 & 0.0469 & 0.0541 & 0.1946 & 0.2183 & 0.248 & 0.2708 \\
6 & 10 & 0.0309 & 0.0346 & 0.0407 & 0.047 & 0.1641 & 0.1831 & 0.2079 & 0.226 \\
6 & 11 & 0.0273 & 0.0305 & 0.0358 & 0.0413 & 0.1422 & 0.1585 & 0.1806 & 0.1951 \\
6 & 12 & 0.0245 & 0.0275 & 0.0322 & 0.0368 & 0.124 & 0.1377 & 0.155 & 0.1676 \\
6 & 13 & 0.0217 & 0.0249 & 0.0292 & 0.0333 & 0.1093 & 0.1218 & 0.1385 & 0.1505 \\
6 & 14 & 0.0205 & 0.0227 & 0.0266 & 0.0303 & 0.0979 & 0.1091 & 0.1231 & 0.1328 \\
6 & 15 & 0.0186 & 0.021 & 0.0247 & 0.0281 & 0.0887 & 0.0979 & 0.111 & 0.1196 \\
6 & 16 & 0.0178 & 0.0196 & 0.0228 & 0.0259 & 0.0804 & 0.0889 & 0.1001 & 0.1087 \\
6 & 17 & 0.0161 & 0.0179 & 0.021 & 0.0239 & 0.0733 & 0.080 & 0.0908 & 0.098 \\
6 & 18 & 0.0154 & 0.0171 & 0.0199 & 0.0225 & 0.0676 & 0.0741 & 0.0829 & 0.089 \\
6 & 19 & 0.0145 & 0.0162 & 0.0187 & 0.0211 & 0.0625 & 0.0688 & 0.0766 & 0.083 \\
6 & 20 & 0.01387 & 0.0152 & 0.0176 & 0.02 & 0.0581 & 0.064 & 0.0714 & 0.0767 \\
6 & 21 & 0.0129 & 0.0143 & 0.0166 & 0.0189 & 0.0542 & 0.0595 & 0.0664 & 0.0713 \\
6 & 22 & 0.0124 & 0.0137 & 0.0158 & 0.0179 & 0.0509 & 0.0559 & 0.0624 & 0.0671 \\
6 & 23 & 0.012 & 0.0133 & 0.0153 & 0.0171 & 0.048 & 0.0527 & 0.0581 & 0.0625 \\
6 & 24 & 0.0114 & 0.0126 & 0.0145 & 0.0163 & 0.0449 & 0.049 & 0.0543 & 0.0582 \\
6 & 25 & 0.011 & 0.0121 & 0.0139 & 0.0156 & 0.0425 & 0.0465 & 0.0514 & 0.055 \\
7 & 7 & 0.056 & 0.0634 & 0.075 & 0.0859 & 0.3162 & 0.3539 & 0.402 & 0.4381 \\
7 & 8 & 0.0455 & 0.0513 & 0.0607 & 0.0696 & 0.2479 & 0.2778 & 0.3174 & 0.3479 \\
7 & 9 & 0.0382 & 0.0431 & 0.0509 & 0.0585 & 0.2059 & 0.2303 & 0.2612 & 0.2853 \\
7 & 10 & 0.0333 & 0.0379 & 0.0446 & 0.0505 & 0.1741 & 0.1934 & 0.2178 & 0.2355 \\
7 & 11 & 0.0291 & 0.033 & 0.0389 & 0.0441 & 0.1488 & 0.1658 & 0.1869 & 0.2029 \\
7 & 12 & 0.0261 & 0.0291 & 0.034 & 0.0389 & 0.1297 & 0.1435 & 0.1618 & 0.1751 \\
\hline
\end{tabular}


Chapter 3. The Two-sample V-statistic for Exponentiality for Complete Samples 35

\begin{tabular}{|cc|cccccccc|}
\hline$n_{1}$ & $n_{2}$ & 0.005 & 0.01 & 0.025 & 0.05 & 0.95 & 0.975 & 0.99 & 0.995 \\
\hline 7 & 14 & 0.0214 & 0.024 & 0.0284 & 0.0323 & 0.1024 & 0.1135 & 0.1276 & 0.1391 \\
7 & 15 & 0.02 & 0.0223 & 0.026 & 0.0295 & 0.0922 & 0.1017 & 0.1145 & 0.1234 \\
7 & 16 & 0.0186 & 0.0207 & 0.024 & 0.0273 & 0.0836 & 0.0919 & 0.1039 & 0.1122 \\
7 & 17 & 0.0167 & 0.019 & 0.0222 & 0.0251 & 0.076 & 0.084 & 0.0941 & 0.102 \\
7 & 18 & 0.0159 & 0.0178 & 0.0208 & 0.0236 & 0.0702 & 0.0771 & 0.0864 & 0.0936 \\
7 & 19 & 0.0151 & 0.0168 & 0.0195 & 0.0221 & 0.0647 & 0.0714 & 0.0794 & 0.085 \\
7 & 20 & 0.0143 & 0.0159 & 0.0184 & 0.0207 & 0.0601 & 0.0657 & 0.073 & 0.0787 \\
7 & 21 & 0.0136 & 0.015 & 0.0174 & 0.0197 & 0.0558 & 0.061 & 0.0679 & 0.0729 \\
7 & 22 & 0.0131 & 0.0145 & 0.0167 & 0.0187 & 0.052 & 0.0569 & 0.0635 & 0.0681 \\
7 & 23 & 0.0123 & 0.0137 & 0.0157 & 0.0177 & 0.0492 & 0.0539 & 0.0592 & 0.0635 \\
7 & 24 & 0.0118 & 0.0131 & 0.0151 & 0.0169 & 0.0463 & 0.0507 & 0.0561 & 0.06 \\
7 & 25 & 0.0115 & 0.0127 & 0.0144 & 0.0162 & 0.0437 & 0.0475 & 0.0524 & 0.0561 \\
8 & 8 & 0.0497 & 0.0557 & 0.0652 & 0.0749 & 0.2637 & 0.2931 & 0.3331 & 0.3636 \\
8 & 9 & 0.0416 & 0.0465 & 0.0551 & 0.0631 & 0.2164 & 0.2412 & 0.2755 & 0.2971 \\
8 & 10 & 0.0359 & 0.0404 & 0.0468 & 0.0537 & 0.1819 & 0.2027 & 0.2292 & 0.2483 \\
8 & 11 & 0.0317 & 0.0356 & 0.0413 & 0.0471 & 0.1542 & 0.1711 & 0.1937 & 0.2128 \\
8 & 12 & 0.0285 & 0.0318 & 0.0369 & 0.0421 & 0.1353 & 0.1502 & 0.1691 & 0.184 \\
8 & 13 & 0.0259 & 0.0283 & 0.0328 & 0.0374 & 0.1191 & 0.1323 & 0.1478 & 0.1596 \\
8 & 14 & 0.023 & 0.0257 & 0.0297 & 0.034 & 0.1064 & 0.1173 & 0.1314 & 0.1415 \\
8 & 15 & 0.0209 & 0.0235 & 0.0276 & 0.0312 & 0.0953 & 0.1049 & 0.1178 & 0.127 \\
8 & 16 & 0.0193 & 0.0215 & 0.0251 & 0.0284 & 0.0863 & 0.0948 & 0.106 & 0.1146 \\
8 & 17 & 0.0181 & 0.0202 & 0.0234 & 0.0264 & 0.0786 & 0.0867 & 0.0966 & 0.1036 \\
8 & 18 & 0.0171 & 0.0189 & 0.022 & 0.0247 & 0.0723 & 0.0797 & 0.0888 & 0.0954 \\
\hline & & & & & & & & &
\end{tabular}


Chapter 3. The Two-sample V-statistic for Exponentiality for Complete Samples 36

\begin{tabular}{|cc|cccccccc|}
\hline$n_{1}$ & $n_{2}$ & 0.005 & 0.01 & 0.025 & 0.05 & 0.95 & 0.975 & 0.99 & 0.995 \\
\hline 8 & 19 & 0.0159 & 0.0177 & 0.0205 & 0.0232 & 0.0666 & 0.0729 & 0.0812 & 0.0868 \\
8 & 20 & 0.0152 & 0.0168 & 0.0194 & 0.0219 & 0.0619 & 0.0677 & 0.0753 & 0.0812 \\
8 & 21 & 0.0143 & 0.0159 & 0.0182 & 0.0204 & 0.0575 & 0.0631 & 0.0703 & 0.0754 \\
8 & 22 & 0.0136 & 0.015 & 0.0173 & 0.0195 & 0.0538 & 0.0588 & 0.0655 & 0.0701 \\
8 & 23 & 0.013 & 0.0144 & 0.0165 & 0.0185 & 0.0502 & 0.0547 & 0.0606 & 0.0651 \\
8 & 24 & 0.0123 & 0.0135 & 0.0156 & 0.0175 & 0.0475 & 0.0519 & 0.0572 & 0.0613 \\
8 & 25 & 0.0117 & 0.013 & 0.015 & 0.0168 & 0.0449 & 0.0489 & 0.0539 & 0.0578 \\
9 & 9 & 0.0446 & 0.0503 & 0.0592 & 0.0675 & 0.2268 & 0.252 & 0.2861 & 0.3129 \\
9 & 10 & 0.0385 & 0.0429 & 0.0501 & 0.0574 & 0.1893 & 0.2092 & 0.2379 & 0.2557 \\
9 & 11 & 0.0336 & 0.0377 & 0.0445 & 0.0504 & 0.1618 & 0.1793 & 0.2023 & 0.2192 \\
9 & 12 & 0.0301 & 0.0337 & 0.0391 & 0.0445 & 0.1408 & 0.1555 & 0.176 & 0.1933 \\
9 & 13 & 0.0268 & 0.0301 & 0.035 & 0.0399 & 0.1236 & 0.1361 & 0.1527 & 0.1655 \\
9 & 14 & 0.0241 & 0.0271 & 0.0317 & 0.0359 & 0.1097 & 0.1211 & 0.1364 & 0.1471 \\
9 & 15 & 0.0228 & 0.0252 & 0.0291 & 0.0329 & 0.0982 & 0.1082 & 0.1213 & 0.1311 \\
9 & 16 & 0.0208 & 0.0229 & 0.0265 & 0.0301 & 0.089 & 0.098 & 0.1094 & 0.1183 \\
9 & 17 & 0.0191 & 0.0211 & 0.0247 & 0.0279 & 0.0812 & 0.0891 & 0.0994 & 0.1079 \\
9 & 18 & 0.0179 & 0.0199 & 0.023 & 0.026 & 0.0742 & 0.0814 & 0.0909 & 0.098 \\
9 & 19 & 0.0169 & 0.0188 & 0.0216 & 0.0243 & 0.0688 & 0.0752 & 0.0836 & 0.0902 \\
9 & 20 & 0.0159 & 0.0176 & 0.0202 & 0.0227 & 0.0636 & 0.0695 & 0.0771 & 0.0824 \\
9 & 21 & 0.0151 & 0.0166 & 0.0191 & 0.0215 & 0.0592 & 0.0645 & 0.0721 & 0.0774 \\
9 & 22 & 0.014 & 0.0157 & 0.0179 & 0.0202 & 0.0553 & 0.06 & 0.0655 & 0.0707 \\
9 & 23 & 0.0136 & 0.015 & 0.0171 & 0.0192 & 0.0518 & 0.0566 & 0.0627 & 0.0672 \\
9 & 24 & 0.013 & 0.0143 & 0.0164 & 0.0183 & 0.0487 & 0.053 & 0.0583 & 0.0627 \\
\hline
\end{tabular}


Chapter 3. The Two-sample V-statistic for Exponentiality for Complete Samples 37

\begin{tabular}{|cc|cccccccc|}
\hline$n_{1}$ & $n_{2}$ & 0.005 & 0.01 & 0.025 & 0.05 & 0.95 & 0.975 & 0.99 & 0.995 \\
\hline 9 & 25 & 0.0124 & 0.0135 & 0.0155 & 0.0174 & 0.0458 & 0.0497 & 0.0548 & 0.0584 \\
10 & 10 & 0.0408 & 0.0461 & 0.054 & 0.0614 & 0.1993 & 0.2207 & 0.2482 & 0.2708 \\
10 & 11 & 0.0354 & 0.0395 & 0.0463 & 0.0529 & 0.1689 & 0.1874 & 0.2122 & 0.2296 \\
10 & 12 & 0.0317 & 0.0352 & 0.0414 & 0.047 & 0.1464 & 0.1619 & 0.1827 & 0.1981 \\
10 & 13 & 0.0283 & 0.0319 & 0.037 & 0.042 & 0.1276 & 0.1413 & 0.1578 & 0.1695 \\
10 & 14 & 0.0257 & 0.0289 & 0.0335 & 0.0379 & 0.1138 & 0.1258 & 0.1413 & 0.1515 \\
10 & 15 & 0.0237 & 0.0263 & 0.0306 & 0.0344 & 0.1021 & 0.1127 & 0.1264 & 0.1373 \\
10 & 16 & 0.0216 & 0.0238 & 0.0277 & 0.0316 & 0.0927 & 0.1015 & 0.1141 & 0.1225 \\
10 & 17 & 0.0202 & 0.0225 & 0.026 & 0.0293 & 0.0835 & 0.0915 & 0.1021 & 0.1097 \\
10 & 18 & 0.0187 & 0.0208 & 0.0239 & 0.027 & 0.0766 & 0.0837 & 0.0934 & 0.0999 \\
10 & 19 & 0.0177 & 0.0197 & 0.0227 & 0.0254 & 0.0707 & 0.0775 & 0.086 & 0.0926 \\
10 & 20 & 0.0167 & 0.0183 & 0.021 & 0.0236 & 0.0655 & 0.0715 & 0.0793 & 0.085 \\
10 & 21 & 0.0157 & 0.0174 & 0.0198 & 0.0223 & 0.0607 & 0.0663 & 0.0735 & 0.0787 \\
10 & 22 & 0.0146 & 0.0163 & 0.0188 & 0.021 & 0.0567 & 0.0619 & 0.0683 & 0.0731 \\
10 & 23 & 0.0142 & 0.0156 & 0.0178 & 0.0198 & 0.0531 & 0.0579 & 0.0637 & 0.0682 \\
10 & 24 & 0.0134 & 0.0148 & 0.0169 & 0.0189 & 0.0497 & 0.0542 & 0.0599 & 0.064 \\
10 & 25 & 0.0126 & 0.0141 & 0.0161 & 0.0179 & 0.047 & 0.0512 & 0.0566 & 0.0606 \\
11 & 11 & 0.0379 & 0.0422 & 0.0493 & 0.0559 & 0.1741 & 0.1928 & 0.2157 & 0.2342 \\
11 & 12 & 0.0335 & 0.0375 & 0.0438 & 0.0495 & 0.1517 & 0.1681 & 0.1881 & 0.2038 \\
11 & 13 & 0.0298 & 0.0331 & 0.0388 & 0.0439 & 0.1328 & 0.1457 & 0.1631 & 0.1766 \\
11 & 14 & 0.0274 & 0.0305 & 0.035 & 0.0396 & 0.118 & 0.1294 & 0.144 & 0.1549 \\
11 & 15 & 0.0249 & 0.0275 & 0.0319 & 0.036 & 0.1051 & 0.1156 & 0.1291 & 0.1383 \\
\hline
\end{tabular}


Chapter 3. The Two-sample V-statistic for Exponentiality for Complete Samples 38

\begin{tabular}{|ll|cccccccc|}
\hline$n_{1}$ & $n_{2}$ & 0.005 & 0.01 & 0.025 & 0.05 & 0.95 & 0.975 & 0.99 & 0.995 \\
\hline 11 & 16 & 0.0227 & 0.0254 & 0.0293 & 0.0329 & 0.0954 & 0.1046 & 0.1157 & 0.124 \\
11 & 17 & 0.021 & 0.0234 & 0.0271 & 0.0304 & 0.0866 & 0.0949 & 0.1052 & 0.1131 \\
11 & 18 & 0.0197 & 0.0216 & 0.025 & 0.0283 & 0.0788 & 0.0865 & 0.0957 & 0.1025 \\
11 & 19 & 0.0185 & 0.0205 & 0.0235 & 0.0264 & 0.0728 & 0.0797 & 0.0886 & 0.0947 \\
11 & 20 & 0.0174 & 0.019 & 0.022 & 0.0247 & 0.067 & 0.0731 & 0.0809 & 0.0867 \\
11 & 21 & 0.0162 & 0.018 & 0.0209 & 0.0234 & 0.0621 & 0.0676 & 0.075 & 0.0798 \\
11 & 22 & 0.0155 & 0.0171 & 0.0197 & 0.0219 & 0.0579 & 0.063 & 0.0691 & 0.074 \\
11 & 23 & 0.0145 & 0.016 & 0.0184 & 0.0206 & 0.0543 & 0.0592 & 0.0652 & 0.0693 \\
11 & 24 & 0.0139 & 0.0154 & 0.0175 & 0.0196 & 0.0509 & 0.0552 & 0.061 & 0.0654 \\
11 & 25 & 0.0135 & 0.0147 & 0.0169 & 0.0187 & 0.0482 & 0.0522 & 0.0575 & 0.0614 \\
12 & 12 & 0.0351 & 0.0395 & 0.0461 & 0.0521 & 0.1564 & 0.1723 & 0.1936 & 0.2088 \\
12 & 13 & 0.0315 & 0.035 & 0.0407 & 0.0459 & 0.1374 & 0.1514 & 0.17 & 0.1824 \\
12 & 14 & 0.0286 & 0.0318 & 0.0369 & 0.0416 & 0.121 & 0.1332 & 0.1489 & 0.1591 \\
12 & 15 & 0.0258 & 0.0284 & 0.0334 & 0.0378 & 0.1087 & 0.1192 & 0.1329 & 0.1424 \\
12 & 16 & 0.0243 & 0.0266 & 0.0306 & 0.0344 & 0.0977 & 0.1069 & 0.1189 & 0.127 \\
12 & 17 & 0.0218 & 0.0245 & 0.0283 & 0.0317 & 0.0887 & 0.0969 & 0.1076 & 0.1155 \\
12 & 18 & 0.0207 & 0.0229 & 0.0263 & 0.0295 & 0.0812 & 0.0888 & 0.0983 & 0.1058 \\
12 & 19 & 0.0193 & 0.0212 & 0.0244 & 0.0273 & 0.0743 & 0.081 & 0.0902 & 0.097 \\
12 & 20 & 0.0182 & 0.02 & 0.0229 & 0.0256 & 0.0688 & 0.0751 & 0.0828 & 0.0884 \\
12 & 21 & 0.017 & 0.0186 & 0.0216 & 0.0242 & 0.0638 & 0.0695 & 0.0769 & 0.0826 \\
12 & 22 & 0.0156 & 0.0174 & 0.0201 & 0.0225 & 0.0593 & 0.0648 & 0.0714 & 0.0765 \\
12 & 23 & 0.0151 & 0.0166 & 0.0191 & 0.0213 & 0.0556 & 0.0607 & 0.0667 & 0.0712 \\
12 & 24 & 0.0146 & 0.016 & 0.0182 & 0.0203 & 0.0522 & 0.0567 & 0.0622 & 0.0661 \\
\hline
\end{tabular}


Chapter 3. The Two-sample V-statistic for Exponentiality for Complete Samples 39

\begin{tabular}{|c|c|c|c|c|c|c|c|c|c|}
\hline$n_{1}$ & $n_{2}$ & 0.005 & 0.01 & 0.025 & 0.05 & 0.95 & 0.975 & 0.99 & 0.995 \\
\hline 12 & 25 & 0.0137 & 0.0151 & 0.0172 & 0.0192 & 0.0491 & 0.0534 & 0.0586 & 0.0626 \\
\hline 13 & 13 & 0.0336 & 0.0373 & 0.0433 & 0.0487 & 0.1417 & 0.1552 & 0.1732 & 0.1853 \\
\hline 13 & 14 & 0.03 & 0.0333 & 0.0387 & 0.0439 & 0.1247 & 0.1372 & 0.1534 & 0.1651 \\
\hline 13 & 15 & 0.0273 & 0.0302 & 0.0349 & 0.0394 & 0.1112 & 0.1218 & 0.1362 & 0.1469 \\
\hline 13 & 16 & 0.0246 & 0.0276 & 0.0317 & 0.0359 & 0.1008 & 0.1102 & 0.1225 & 0.132 \\
\hline 13 & 17 & 0.0228 & 0.0252 & 0.0291 & 0.0329 & 0.0914 & 0.0996 & 0.1101 & 0.1185 \\
\hline 13 & 18 & 0.0214 & 0.0235 & 0.0272 & 0.0305 & 0.0832 & 0.0907 & $0.101^{\circ}$ & 0.108 \\
\hline 13 & 19 & 0.0199 & 0.022 & 0.0253 & 0.0284 & 0.0763 & 0.0831 & 0.0918 & 0.099 \\
\hline 13 & 20 & 0.0185 & 0.0204 & 0.0236 & 0.0264 & 0.0705 & 0.0769 & 0.0854 & 0.0914 \\
\hline 13 & 21 & 0.0176 & 0.0195 & 0.0224 & 0.0249 & 0.0652 & 0.0711 & 0.0787 & 0.0845 \\
\hline 13 & 22 & 0.0167 & 0.0185 & 0.021 & 0.0235 & 0.061 & 0.0663 & 0.0735 & 0.0781 \\
\hline 13 & 23 & 0.0157 & 0.0174 & 0.0199 & 0.0222 & 0.0571 & 0.0619 & 0.0683 & 0.0725 \\
\hline 13 & 24 & 0.015 & 0.0165 & 0.0187 & 0.0209 & 0.0535 & 0.058 & 0.0638 & 0.0685 \\
\hline 13 & 25 & 0.0142 & 0.0157 & 0.0178 & 0.0198 & 0.0503 & 0.0545 & 0.0599 & 0.0639 \\
\hline 14 & 14 & 0.0318 & 0.0349 & 0.0403 & 0.0454 & 0.1292 & 0.1411 & 0.156 & 0.1671 \\
\hline 14 & 15 & 0.0285 & 0.0315 & 0.0364 & 0.0411 & 0.115 & 0.1258 & 0.1398 & 0.1493 \\
\hline 14 & 16 & 0.0262 & 0.0291 & 0.0335 & 0.0376 & 0.1035 & 0.1129 & 0.1265 & 0.137 \\
\hline 14 & 17 & 0.0239 & 0.0266 & 0.0308 & 0.0344 & 0.0938 & 0.1022 & 0.1131 & 0.1201 \\
\hline 14 & 18 & 0.0224 & 0.0246 & 0.0284 & 0.0318 & 0.0851 & 0.0929 & 0.1027 & 0.1094 \\
\hline 14 & 19 & 0.0209 & 0.0231 & 0.0265 & 0.0296 & 0.0786 & 0.0859 & 0.0949 & 0.1008 \\
\hline 14 & 20 & 0.0196 & 0.0214 & 0.0247 & 0.0277 & 0.0723 & 0.0788 & 0.0872 & 0.0933 \\
\hline 14 & 21 & 0.0185 & 0.0202 & 0.0231 & 0.0258 & 0.0671 & 0.0729 & 0.0804 & 0.0862 \\
\hline
\end{tabular}


Chapter 3. The Two-sample V-statistic for Exponentiality for Complete Samples 40

\begin{tabular}{|ll|cccccccc|}
\hline$n_{1}$ & $n_{2}$ & 0.005 & 0.01 & 0.025 & 0.05 & 0.95 & 0.975 & 0.99 & 0.995 \\
\hline 14 & 22 & 0.0173 & 0.019 & 0.0217 & 0.0243 & 0.0624 & 0.0677 & 0.0744 & 0.0792 \\
14 & 23 & 0.0162 & 0.0177 & 0.0202 & 0.0226 & 0.0584 & 0.0634 & 0.0696 & 0.0739 \\
14 & 24 & 0.0154 & 0.017 & 0.0194 & 0.0216 & 0.0546 & 0.0589 & 0.0649 & 0.0694 \\
14 & 25 & 0.0149 & 0.0163 & 0.0185 & 0.0205 & 0.0512 & 0.0554 & 0.0612 & 0.0653 \\
15 & 15 & 0.0302 & 0.0333 & 0.0383 & 0.0428 & 0.1185 & 0.1296 & 0.1432 & 0.1536 \\
15 & 16 & 0.0272 & 0.03 & 0.0351 & 0.0392 & 0.107 & 0.1169 & 0.13 & 0.1391 \\
15 & 17 & 0.0253 & 0.0278 & 0.0321 & 0.0358 & 0.0964 & 0.1051 & 0.117 & 0.1254 \\
15 & 18 & 0.0234 & 0.0257 & 0.0296 & 0.033 & 0.0876 & 0.0952 & 0.1049 & 0.1123 \\
15 & 19 & 0.0217 & 0.024 & 0.0273 & 0.0305 & 0.0803 & 0.0875 & 0.0969 & 0.1029 \\
15 & 20 & 0.0202 & 0.0221 & 0.0255 & 0.0283 & 0.0742 & 0.0804 & 0.0886 & 0.095 \\
15 & 21 & 0.0187 & 0.0207 & 0.0236 & 0.0265 & 0.0685 & 0.0745 & 0.0816 & 0.087 \\
15 & 22 & 0.0179 & 0.0197 & 0.0224 & 0.0249 & 0.0638 & 0.0691 & 0.076 & 0.0811 \\
15 & 23 & 0.0168 & 0.0185 & 0.0212 & 0.0236 & 0.0593 & 0.0642 & 0.0702 & 0.0746 \\
15 & 24 & 0.0162 & 0.0177 & 0.02 & 0.0222 & 0.0557 & 0.0604 & 0.0662 & 0.0706 \\
15 & 25 & 0.0155 & 0.017 & 0.0191 & 0.0211 & 0.0523 & 0.0567 & 0.0623 & 0.0659 \\
16 & 16 & 0.0283 & 0.0314 & 0.0359 & 0.0404 & 0.109 & 0.1188 & 0.1315 & 0.1407 \\
16 & 17 & 0.0263 & 0.0285 & 0.0329 & 0.0371 & 0.0986 & 0.1074 & 0.1186 & 0.1276 \\
16 & 18 & 0.0243 & 0.0268 & 0.0305 & 0.0341 & 0.09 & 0.0981 & 0.1075 & 0.1151 \\
16 & 19 & 0.0224 & 0.0247 & 0.0283 & 0.0316 & 0.082 & 0.0892 & 0.0979 & 0.1049 \\
16 & 20 & 0.021 & 0.023 & 0.0262 & 0.0293 & 0.0759 & 0.0821 & 0.0906 & 0.0964 \\
16 & 21 & 0.0197 & 0.0216 & 0.0246 & 0.0274 & 0.0699 & 0.076 & 0.0835 & 0.0884 \\
16 & 22 & 0.0186 & 0.0204 & 0.0233 & 0.0259 & 0.0651 & 0.071 & 0.0777 & 0.0827 \\
\hline
\end{tabular}


Chapter 3. The Two-sample V-statistic for Exponentiality for Complete Samples 41

\begin{tabular}{|cc|cccccccc|}
\hline$n_{1}$ & $n_{2}$ & 0.005 & 0.01 & 0.025 & 0.05 & 0.95 & 0.975 & 0.99 & 0.995 \\
\hline 16 & 23 & 0.0174 & 0.0191 & 0.0219 & 0.0242 & 0.0609 & 0.0662 & 0.0724 & 0.0774 \\
16 & 24 & 0.0166 & 0.018 & 0.0206 & 0.023 & 0.0567 & 0.0613 & 0.0671 & 0.0717 \\
16 & 25 & 0.0157 & 0.0172 & 0.0195 & 0.0217 & 0.0533 & 0.0574 & 0.0626 & 0.066 \\
17 & 17 & 0.0271 & 0.03 & 0.0343 & 0.0383 & 0.101 & 0.1101 & 0.1214 & 0.1293 \\
17 & 18 & 0.0247 & 0.0275 & 0.0315 & 0.0351 & 0.0922 & 0.1004 & 0.111 & 0.1182 \\
17 & 19 & 0.0234 & 0.0255 & 0.0292 & 0.0325 & 0.084 & 0.0914 & 0.1007 & 0.1074 \\
17 & 20 & 0.0219 & 0.024 & 0.0274 & 0.0306 & 0.0773 & 0.0838 & 0.0921 & 0.0977 \\
17 & 21 & 0.0205 & 0.0225 & 0.0255 & 0.0284 & 0.0713 & 0.0774 & 0.085 & 0.0907 \\
17 & 22 & 0.0193 & 0.0211 & 0.0239 & 0.0266 & 0.0664 & 0.0718 & 0.0786 & 0.084 \\
17 & 23 & 0.018 & 0.0196 & 0.0224 & 0.0249 & 0.0619 & 0.0668 & 0.0738 & 0.0787 \\
17 & 24 & 0.0172 & 0.0189 & 0.0212 & 0.0236 & 0.0578 & 0.0625 & 0.0684 & 0.073 \\
17 & 25 & 0.0163 & 0.0179 & 0.0202 & 0.0224 & 0.0545 & 0.0593 & 0.0647 & 0.069 \\
18 & 18 & 0.0262 & 0.0286 & 0.0327 & 0.0365 & 0.094 & 0.1018 & 0.1121 & 0.1198 \\
18 & 19 & 0.0241 & 0.0264 & 0.0304 & 0.0338 & 0.0861 & 0.0935 & 0.1031 & 0.1095 \\
18 & 20 & 0.0223 & 0.0245 & 0.028 & 0.0314 & 0.0791 & 0.0856 & 0.0943 & 0.1003 \\
18 & 21 & 0.0209 & 0.0229 & 0.0262 & 0.0292 & 0.0733 & 0.0794 & 0.0873 & 0.0937 \\
18 & 22 & 0.0199 & 0.0218 & 0.0247 & 0.0273 & 0.0682 & 0.0735 & 0.081 & 0.0868 \\
18 & 23 & 0.0186 & 0.0205 & 0.0233 & 0.0257 & 0.0632 & 0.0685 & 0.0752 & 0.08 \\
18 & 24 & 0.0179 & 0.0194 & 0.0219 & 0.0243 & 0.059 & 0.0639 & 0.0699 & 0.0744 \\
18 & 25 & 0.0166 & 0.0183 & 0.0208 & 0.0229 & 0.0555 & 0.0601 & 0.0657 & 0.0699 \\
19 & 19 & 0.0248 & 0.0275 & 0.0313 & 0.0349 & 0.0882 & 0.0958 & 0.1055 & 0.1128 \\
19 & 20 & 0.0231 & 0.0254 & 0.029 & 0.0322 & 0.0807 & 0.0874 & 0.0954 & 0.1008 \\
\hline
\end{tabular}


Chapter 3. The Two-sample V-statistic for Exponentiality for Complete Samples 42

\begin{tabular}{|ll|cccccccc|}
\hline$n_{1}$ & $n_{2}$ & 0.005 & 0.01 & 0.025 & 0.05 & 0.95 & 0.975 & 0.99 & 0.995 \\
\hline 19 & 21 & 0.0217 & 0.0239 & 0.0271 & 0.0303 & 0.0749 & 0.0811 & 0.0886 & 0.094 \\
19 & 22 & 0.0203 & 0.0223 & 0.0254 & 0.0282 & 0.0693 & 0.0748 & 0.082 & 0.0868 \\
19 & 23 & 0.0192 & 0.0211 & 0.0238 & 0.0264 & 0.0646 & 0.0697 & 0.0759 & 0.0808 \\
19 & 24 & 0.018 & 0.0196 & 0.0224 & 0.0249 & 0.0603 & 0.0653 & 0.0713 & 0.0763 \\
19 & 25 & 0.017 & 0.0187 & 0.0213 & 0.0236 & 0.0567 & 0.0611 & 0.0665 & 0.0705 \\
20 & 20 & 0.0241 & 0.0265 & 0.03 & 0.0333 & 0.0823 & 0.089 & 0.0972 & 0.1042 \\
20 & 21 & 0.0226 & 0.0247 & 0.0281 & 0.0311 & 0.0762 & 0.0823 & 0.0901 & 0.0957 \\
20 & 22 & 0.021 & 0.0231 & 0.0261 & 0.0289 & 0.0706 & 0.0764 & 0.0839 & 0.0892 \\
20 & 23 & 0.0196 & 0.0216 & 0.0245 & 0.0272 & 0.0659 & 0.071 & 0.0778 & 0.0827 \\
20 & 24 & 0.0186 & 0.0204 & 0.0232 & 0.0256 & 0.0615 & 0.0662 & 0.0726 & 0.0766 \\
20 & 25 & 0.0176 & 0.0194 & 0.0219 & 0.0242 & 0.0575 & 0.0619 & 0.0675 & 0.0718 \\
21 & 21 & 0.0233 & 0.0256 & 0.0289 & 0.032 & 0.0778 & 0.084 & 0.0921 & 0.0984 \\
21 & 22 & 0.0221 & 0.024 & 0.0271 & 0.0299 & 0.0723 & 0.0779 & 0.0852 & 0.0902 \\
21 & 23 & 0.0203 & 0.0223 & 0.0252 & 0.0279 & 0.0669 & 0.0723 & 0.0791 & 0.0837 \\
21 & 24 & 0.0193 & 0.0211 & 0.0239 & 0.0264 & 0.0626 & 0.0674 & 0.0737 & 0.0779 \\
21 & 25 & 0.0182 & 0.02 & 0.0226 & 0.0249 & 0.0587 & 0.063 & 0.0691 & 0.073 \\
22 & 22 & 0.0222 & 0.0244 & 0.0277 & 0.0306 & 0.0736 & 0.0796 & 0.0866 & 0.0922 \\
22 & 23 & 0.0212 & 0.0231 & 0.026 & 0.0287 & 0.0685 & 0.0738 & 0.0805 & 0.0855 \\
22 & 24 & 0.0199 & 0.0217 & 0.0244 & 0.027 & 0.0638 & 0.0687 & 0.0748 & 0.079 \\
22 & 25 & 0.019 & 0.0205 & 0.0231 & 0.0254 & 0.0597 & 0.064 & 0.0697 & 0.0739 \\
23 & 23 & 0.0216 & 0.0236 & 0.0266 & 0.0296 & 0.0696 & 0.075 & 0.0821 & 0.0869 \\
23 & 24 & 0.0204 & 0.0224 & 0.0251 & 0.0277 & 0.0649 & 0.0699 & 0.0764 & 0.0808 \\
\hline & & & & & & & & & \\
\hline
\end{tabular}


Chapter 3. The Two-sample V-statistic for Exponentiality for Complete Samples 43

\begin{tabular}{|cc|cccccccc|}
\hline$n_{1}$ & $n_{2}$ & 0.005 & 0.01 & 0.025 & 0.05 & 0.95 & 0.975 & 0.99 & 0.995 \\
\hline 23 & 25 & 0.0194 & 0.0211 & 0.0237 & 0.0261 & 0.0607 & 0.0651 & 0.071 & 0.0752 \\
24 & 24 & 0.0211 & 0.023 & 0.0258 & 0.0285 & 0.0661 & 0.0711 & 0.0776 & 0.0822 \\
24 & 25 & 0.0199 & 0.0218 & 0.0244 & 0.0269 & 0.0619 & 0.0666 & 0.0726 & 0.0763 \\
25 & 25 & 0.0204 & 0.0222 & 0.0249 & 0.0274 & 0.0627 & 0.0674 & 0.074 & 0.0779 \\
\hline
\end{tabular}

\subsection{Numerical Example}

\section{EXAMPLE 1 .}

Proschan (1963) has given the number of successive failures of air-conditioning system of each member of a fleet of 13 Boeing 720 jet airplanes. The hours of flying time between failures are listed below for two of the planes.

Plane 7908: 413, 14, 58, 37, 100, 65, 9, 169, 447, 184, 36, 201, 118.

Plane 7911: 55, 320, 56, 104, 220, 239, 47, 246, 176, 182, 33.

For testing the null hypothesis $H_{10}$

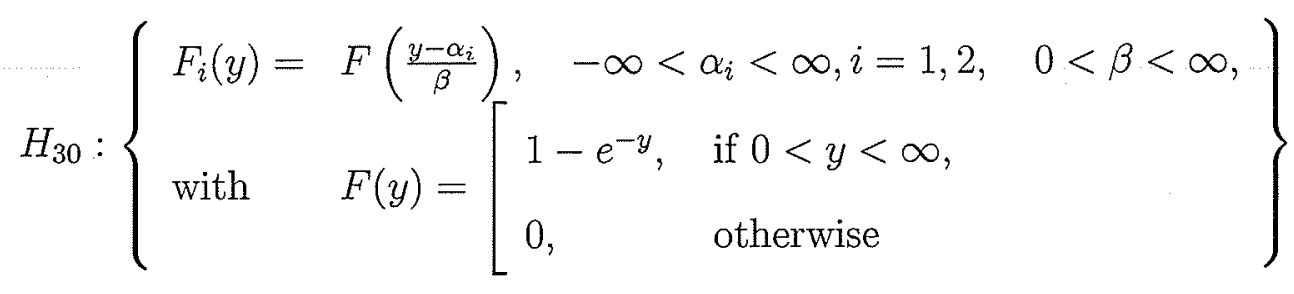


Chapter 3. The Two-sample V-statistic for Exponentiality for Complete Samples 44 against the alternative hypothesis

$$
H_{3 a}:\left\{\begin{array}{ll}
F_{i}(y)= & F\left(\frac{y-\alpha_{i}}{\beta}\right) \\
\text { with } \quad F(y) \neq 1-e^{-y}, \quad \text { for some } y
\end{array}\right\}
$$

In this example, we have

$$
\begin{aligned}
n_{1} & =13, n_{2}=11, r_{1}=s_{1}=r_{2}=s_{2}=0 \\
\bar{y}_{1} & =\frac{1}{13} \sum_{j=1}^{13} y_{1 j} \\
& =142.385 \\
\bar{y}_{2} & =\frac{1}{11} \sum_{j=1}^{11} y_{2 j} \\
& =152.545, \\
S_{1}^{2} & =\sum_{j=1}^{13}\left(y_{1 j}-\bar{y}_{1}\right)^{2} \\
& =244097.0769, \\
S_{2}^{2} & =\sum_{j=1}^{11}\left(y_{2 j}-\bar{y}_{2}\right)^{2} \\
& =96840.72727, \\
S^{2} & =S_{1}^{2}+S_{2}^{2} \\
& =244097.0769+96840.72727 \\
& =340937.8042 \\
n^{*} & =\max (13,11)=13 .
\end{aligned}
$$


Chapter 3. The Two-sample V-statistic for Exponentiality for Complete Samples 45

Hence we calculate the V-exponential statistic as following:

$$
\begin{aligned}
V\left(n_{1}, n_{2}\right) & =\frac{\left\{n_{1}\left(\bar{Y}_{1}-Y_{11}\right)+n_{2}\left(\bar{Y}_{2}-Y_{21}\right)\right\}^{2}}{2 n^{*}\left(n^{*}-1\right) S^{2}} \\
V(13,11) & =\frac{\{13(142.385-9)+11(152.545-33)\}^{2}}{2 \times 13 \times(13-1) \times 340937.8042} \\
& =0.0874 .
\end{aligned}
$$

From Table 3.1, it is seen that this value is greater than the lower $5 \%$ value 0.0439 corresponding to $n_{1}=13$ and $n_{2}=11$. Therefore, we conclude that there is no evidence of non-exponentiality of the two failure distributions with equal but unknown location parameters and same unknown scale parameters. 


\section{Chapter 4}

\section{Sensitivity Results for the Two-sample V-statistic}

We used the empirical sampling results from Table 3.1 to evaluate the sensitivity properties of theV-exponential statistic for exponentiality for various alternative distributions.

As we mentioned before, the V-exponential statistic responds to nonexponentiality by assuming either small or large values, so that the test needs to be two-tailed in general. That is, for each pair of $n_{1}$ and $n_{2}$ (i.e. $n_{1}=n_{2}=5, n_{1}=n_{2}=10$, $n_{1}=n_{2}=15, n_{1}=n_{2}=20, n_{1}=n_{2}=25$ ), we calculate the proportions which fell above the $95 \%$ point and below the $5 \%$ point for the alternative distributions. The sum of these two proportions provides the empirical power of a two-tailed test with $10 \%$ significance level. The choice of the probability distribution under the alternative hypothesis was based on the distributions commonly used as alternatives to the exponential distribution (i.e. the Weibull, $\chi^{2}$, half-normal, and lognormal dis-

tributions) and the distributions with $\mathrm{U}$-shape hazard functions (i.e. power function distributions). For the functional forms of the density functions of these distributions, see Brain and Shapiro (1983).

As expected, the $V$-exponential statistic is quite sensitive to departures form exponentiality and especially to symmetric alternative distribution. The estimated power 
increases as the two sample sizes increase, and is greater than the corresponding estimated power of the one sample $\mathrm{W}$-exponential test at the $10 \%$ significance level for $n=5,10,15,20,25$ (see Table 3 of Shapiro and Wilk, 1972). For example, for $n_{1}=n_{2}=15$, the estimated power of the $\mathrm{V}$-exponential test is quite close to the corresponding estimate for the one sample $\mathrm{W}$-exponential test at the $10 \%$ significance level for $n=30$ (see Table 1 of Samanta and Schwarz, 1988). The powers are given in Table 4.1. 
Chapter 4. Sensitivity Results for the Two-sample V-statistic

Table 4.1: Estimated Powers of the V-exponential test for selected sample sizes at $10 \%$ significance level.

\begin{tabular}{|c|c|c|c|c|c|}
\hline & $n_{1}=n_{2}=5$ & $n_{1}=n_{2}=10$ & $n_{1}=n_{2}=15$ & $n_{1}=n_{2}=20$ & $n_{1}=n_{2}=25$ \\
\hline$\chi^{2}(1)$ & 0.2044 & 0.3492 & 0.5024 & 0.5902 & 0.706 \\
$\chi^{2}(4)$ & 0.1198 & 0.2488 & 0.3672 & 0.5002 & 0.6062 \\
$\chi^{2}(6)$ & 0.1582 & 0.386 & 0.5992 & 0.7682 & 0.868 \\
Beta(1,3) & 0.1188 & 0.226 & 0.348 & 0.4914 & 0.5892 \\
Lognormal(0,0.3) & 0.2216 & 0.583 & 0.8032 & 0.9214 & 0.9634 \\
Lognormal(0,0.7) & 0.122 & 0.165 & 0.1962 & 0.2376 & 0.2608 \\
Lognormal(0,1) & 0.184 & 0.2716 & 0.3474 & 0.4114 & 0.465 \\
Weibull(2.0,1) & 0.2466 & 0.6278 & 0.8688 & 0.968 & 0.9908 \\
Weibull(0.5,1) & 0.4622 & 0.7766 & 0.9146 & 0.9694 & 0.9886 \\
Half-normal & 0.13 & 0.257 & 0.4144 & 0.5672 & 0.6618 \\
Half-Cauchy & 0.4848 & 0.7218 & 0.862 & 0.9284 & 0.9616 \\
Uniform(0, 1) & 0.5888 & 0.7876 & 0.9568 & 0.9902 & 0.9992 \\
Normal & 0.8488 & 0.8946 & 0.9896 & 0.9994 & 0.9999 \\
t-distribution(2) & 0.4534 & 0.8 & 0.8988 & 0.9408 & 0.9598 \\
t-distribution(3) & 0.4414 & 0.8406 & 0.9392 & 0.9714 & 0.988 \\
t-distribution(4) & 0.4432 & 0.8594 & 0.9562 & 0.9866 & 0.9962 \\
Power Function(1/5) & 0.3708 & 0.6148 & 0.7764 & 0.8828 & 0.9376 \\
Power Function(1/3) & 0.1038 & 0.1184 & 0.131 & 0.1438 & 0.1666 \\
Power Function(1/2) & 0.1088 & 0.1314 & 0.1504 & 0.1828 & 0.2094 \\
\hline
\end{tabular}




\section{Chapter 5}

\section{The Two-sample V-statistic for Censored Samples}

Now suppose that, in a random sample of size $n_{1}$ from the distribution function $F_{1}(y)$ the $r_{1}$ smallest and the $r_{2}$ largest observations are censored. Therefore we have the $n_{1}-r_{1}-r_{2}$ middle observations $Y_{1, r_{1}+1} \leq Y_{1, r_{1}+2} \leq \ldots \leq Y_{1, n_{1}-r_{2}}$ available. Further suppose that in another random sample of size $n_{2}$ (first and second sample are independent) from $F_{2}(y)$ and similarly to the first sample, the $s_{1}$ smallest and the $s_{2}$ largest observations are censored. Therefore we have the $n_{2}-s_{1}-s_{2}$ middle observations $Y_{2, s_{1}+1} \leq Y_{2, s_{1}+2} \leq \ldots \leq Y_{2, n_{2}-s_{2}}$ available from the second sample.

Now we define

$$
\begin{aligned}
& q_{1}=\left(n_{1}-r_{1}-r_{2}\right), \\
& q_{2}=\left(n_{2}-s_{1}-s_{2}\right), \\
& q^{*}=\max \left(q_{1}, q_{2}\right), \\
& A=\frac{\sum_{i=2}^{q_{1}} \sum_{j=2}^{q_{1}} a_{i j}^{\left(q_{1}\right)} T_{1, r_{1}+i} T_{1, r_{1}+j}}{q_{1}}, \\
& B=\frac{\sum_{i=2}^{q_{2}} \sum_{j=2}^{q_{2}} b_{i j}^{\left(q_{2}\right)} T_{2, s_{1}+i} T_{2, s_{1}+j}}{q_{2}},
\end{aligned}
$$

and

$$
a_{i j}^{\left(q_{1}\right)}=\frac{(j-1)}{\left(q_{1}-j+1\right)}, \quad\left(q_{1} \geq i \geq j \geq 2\right)
$$


Chapter 5. The Two-sample V-statistic for Censored Samples

$$
b_{i j}^{\left(q_{2}\right)}=\frac{(j-1)}{\left(q_{2}-j+1\right)}, \quad\left(q_{2} \geq i \geq j \geq 2\right) .
$$

with $a_{i j}^{\left(q_{1}\right)}=a_{j i}^{\left(q_{1}\right)}$ and $b_{i j}^{\left(q_{2}\right)}=b_{j i}^{\left(q_{2}\right)}$ as defined before.

Then, using the V-exponential statistic for the uncensored data

$$
V\left(n_{1}, n_{2}\right)=\frac{\left\{n_{1}\left(\bar{Y}_{1}-Y_{11}\right)+n_{2}\left(\bar{Y}_{2}-Y_{21}\right)\right\}^{2}}{2 n^{*}\left(n^{*}-1\right) S^{2}}
$$

we propose a statistic $V_{1}$ for the null hypothesis:

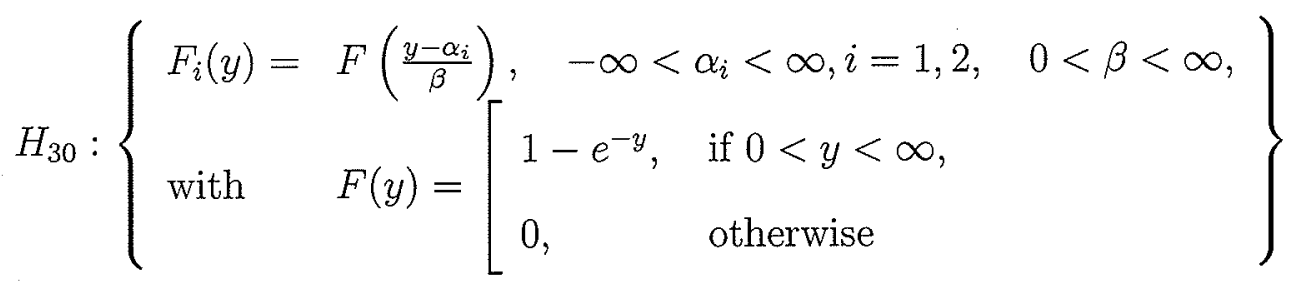

against the alternative hypothesis

$$
H_{3 a}:\left\{\begin{array}{ll}
F_{i}(y)= & F\left(\frac{y-\alpha_{i}}{\beta}\right) \\
\text { with } \quad & F(y) \neq 1-e^{-y}, \quad \text { for some } y
\end{array}\right\}
$$

as defined by

$$
V_{1}=\frac{\left\{\sum_{i=2}^{q_{1}} T_{1, r_{1}+i}+\sum_{i=2}^{q_{2}} T_{2, s_{1}+i}\right\}^{2}}{2 q^{*}\left(q^{*}-1\right)(A+B)} .
$$


Note that

- the null distribution of $V_{1}$ is the same as that of $V\left(q_{1}, q_{2}\right)$,

- $V_{1}$ and $V\left(n_{1}, n_{2}\right)$ are equal when there are no censored observations.

For convenience in computation, we further define

$$
\begin{gathered}
C=\frac{\sum_{i=2}^{q_{1}} \frac{i-1}{q_{1}-i+1} T_{1, r_{1}+i}\left(T_{1, r_{1}+i}+2 \sum_{j=i+1}^{q_{1}} T_{1, r_{1}+j}\right)}{q_{1}}, \\
D=\frac{\sum_{i=2}^{q_{2}} \frac{i-1}{q_{2}-i+1} T_{2, s_{1}+i}\left(T_{2, s_{1}+i}+2 \sum_{j=i+1}^{q_{2}} T_{2, s_{1}+j}\right)}{q_{2}},
\end{gathered}
$$

and point out that we can use the following expression of $V_{1}$

$$
V_{1}=\frac{\left\{\sum_{i=2}^{q_{1}} T_{1, r_{1}+i}+\sum_{i=2}^{q_{2}} T_{2, s_{1}+i}\right\}^{2}}{2 q^{*}\left(q^{*}-1\right)(C+D)} .
$$

EXAMPLE 2 .

We consider the data in Bain (1978) (Problem number 4, page 203). In a new process of making tires a certain additive is proposed for increasing the length of 
time of tread wear of a tire. 40 of the present tires and 40 tires made under the new process are placed in service and the experiment is continued until the 20 smallest observations (in thousands of miles) are obtained for each sample. [The data are not reported here].

In this example, $n_{1}=n_{2}=40, r_{1}=s_{1}=0$ and $r_{2}=s_{2}=20$. From the data, the computed value of $V_{1}$ for testing the null hypothesis $H_{30}$ is $V_{1}=0.0332$.

From Table 3.1, one finds this value to be greater than the lower $2.5 \%$ point of the (null) distribution of $V(20,20)$ and very close to the $5 \%$ point of this distribution. Since both tails of this null distribution are used as critical regions defined by equal tail areas, there is no evidence against the null hypothesis $H_{30}$ and the data do not refute the exponentiality of both distributions with equal but unknown wearing rates. 


\section{Chapter 6}

\section{Two-sample $V^{*}$-statistic for Exponentiality for Complete Samples}

In this chapter, we propose another test statistic for testing exponentiality of two distributions using the one-sample approach of Stephens (1978). We show that our proposed statistic has the same null distribution as Shapiro and Wilk statistic with an appropriate sample size. We also provide an example to illustrate the application of the proposed method.

\section{1 $V^{*}$-exponential statistic for complete sample}

Suppose $Y_{11} \leq Y_{12} \leq \ldots \leq Y_{1 n_{1}}$ are the order statistics of a random sample of size $n_{1}$ from a continuous distribution function $F_{1}(y)$ and $Y_{21} \leq Y_{22} \leq \ldots \leq Y_{2 n_{2}}$ are the order statistics of a random sample of size $n_{2}$ from another continuous distribution function $F_{2}(y)$. Further suppose that the two samples are independent of each other. On the basis of these, we wish to test the null hypothesis:

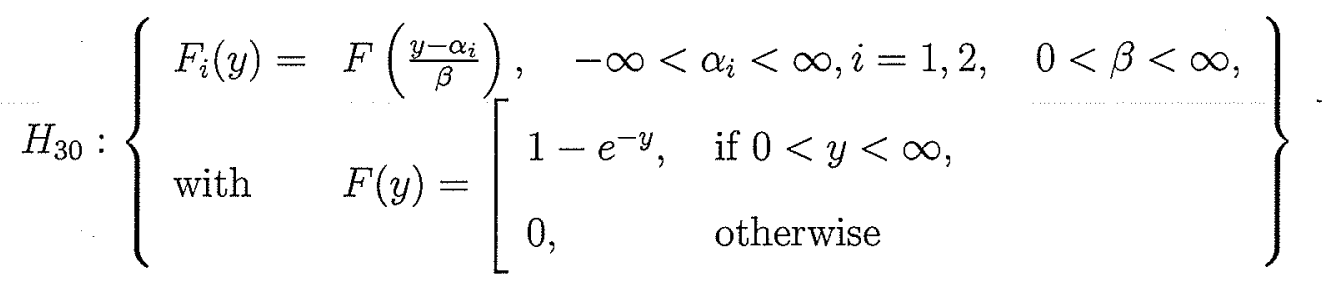


against the alternative hypothesis

$$
H_{3 a}:\left\{\begin{array}{ll}
F_{i}(y)= & F\left(\frac{y-\alpha_{i}}{\beta}\right) \\
\text { with } & F(y) \neq 1-e^{-y}, \quad \text { for some } y
\end{array}\right\}
$$

We define

$$
\begin{aligned}
\bar{Y}_{i} & =\frac{1}{n_{i}} \sum_{j=1}^{n_{i}} Y_{i j}, \quad i=1,2, \\
S_{i}^{2} & =\sum_{j=1}^{n_{i}}\left(Y_{i j}-\bar{Y}_{i}\right)^{2}, \quad i=1,2, \\
X_{i j} & =Y_{i j}-Y_{i 1}, \quad j=1,2, \cdots, n_{i}, \quad i=1,2,
\end{aligned}
$$

Then, we have that

$$
\begin{aligned}
X_{i 1} & =0, \quad i=1,2 \\
\sum_{j=2}^{n_{i}} X_{i j} & =n_{i}\left(\bar{Y}_{i}-Y_{i 1}\right), \quad i=1,2, \\
X_{i j}-\bar{X}_{i} & =Y_{i j}-\bar{Y}_{i}, \quad j=1,2, \cdots, n_{i}, \quad i=1,2,
\end{aligned}
$$

where

$$
\bar{X}_{i}=\frac{1}{n_{i}} \sum_{j=2}^{n_{i}} X_{i j}, \quad i=1,2
$$

From this, we can also write 
Chapter 6. Two-sample $V^{*}$-statistic for Exponentiality for Complete Samples

$$
S_{i}^{2}=\sum_{j=2}^{n_{i}}\left(X_{i j}-\bar{X}_{i}\right)^{2}, \quad i=1,2
$$

and

$$
\begin{aligned}
\left(\bar{Y}_{i}-Y_{i 1}\right)^{2} & =\bar{X}_{i}^{2} \\
& =\frac{1}{n_{i}^{2}}\left(\sum_{j=2}^{n_{i}} X_{i j}\right)^{2}, \quad i=1,2 .
\end{aligned}
$$

We note that under the null hypothesis $H_{30}, X_{i 2} \leq X_{i 3} \leq \ldots \leq X_{i n_{i}}$ are the order statistics of a random sample of size $n_{i}-1$ from the exponential distribution function

$$
G(x)= \begin{cases}1-e^{-x / \beta}, & 0<\beta<\infty, \quad 0<x<\infty \\ 0, & \text { otherwise }\end{cases}
$$

Clearly, under the null hypothesis $H_{30}$, these two samples can be combined to form a single random sample of size $n_{1}+n_{2}-2$ from the above exponential distribution $G(x)$.

If $Z_{1} \leq Z_{2} \leq \ldots \leq Z_{n_{1}+n_{2}-2}$ are the order statistics of this combined sample, then following Stephens (1978), we propose a two sample test statistic $V^{*}\left(n_{1}, n_{2}\right)$ given by 
Chapter 6. Two-sample $V^{*}$-statistic for Exponentiality for Complete Samples

$$
\begin{aligned}
V^{*}\left(n_{1}, n_{2}\right) & =\frac{\left(\sum_{i=1}^{n_{1}+n_{2}-2} Z_{i}\right)^{2}}{\left(n_{1}+n_{2}-2\right)\left\{\left(n_{1}+n_{2}-1\right) \sum_{i=1}^{n_{1}+n_{2}-2} Z_{i}^{2}-\left(\sum_{i=1}^{n_{1}+n_{2}-2} Z_{i}\right)^{2}\right\}} \\
& =\frac{\left(\sum_{i=1}^{2} \sum_{j=2}^{n_{i}} X_{i j}\right)^{2}}{\left\{\left(n_{1}+n_{2}-2\right)\left\{\left(n_{1}+n_{2}-1\right)\left[\sum_{i=1}^{2} \sum_{j=2}^{n_{i}} X_{i j}^{2}\right]-\left(\sum_{i=1}^{2} \sum_{j=2}^{n_{i}} X_{i j}\right)^{2}\right\}\right.} \\
& =\frac{\left\{n_{1}\left(\bar{Y}_{1}-Y_{11}\right)+n_{2}\left(\bar{Y}_{2}-Y_{21}\right)\right\}^{2}}{\left(n_{1}+n_{2}-2\right)\left\{\left(n_{1}+n_{2}-1\right)\left[S_{1}^{2}+S_{2}^{2}\right]-\left[n_{1}\left(\bar{Y}_{1}-Y_{11}\right)+n_{2}\left(\bar{Y}_{2}-Y_{21}\right)\right]^{2}\right\}} .
\end{aligned}
$$

Under the null hypothesis $H_{30}, V^{*}\left(n_{1}, n_{2}\right)$ has the same distribution as the null distribution of the statistic $W_{E}\left(n_{1}+n_{2}-1\right)$ proposed by Shapiro and Wilk (1972).

We note that the above statistic $V^{*}\left(n_{1}, n_{2}\right)$ may be regarded as a two-sample generalization of the one-sample Shapiro-Wilk statistic.

\subsection{Numerical Example}

We revisit EXAMPLE 1 from Chapter 3. 
Here the test statistic is

$$
V^{*}\left(n_{1}, n_{2}\right)=\frac{\left\{n_{1}\left(\bar{Y}_{1}-Y_{11}\right)+n_{2}\left(\bar{Y}_{2}-Y_{21}\right)\right\}^{2}}{\left(n_{1}+n_{2}-2\right)\left\{\left(n_{1}+n_{2}-1\right)\left[S_{1}^{2}+S_{2}^{2}\right]-\left[n_{1}\left(\bar{Y}_{1}-Y_{11}\right)+n_{2}\left(\bar{Y}_{2}-Y_{21}\right)\right]^{2}\right\}}
$$

where

$$
\begin{aligned}
\bar{Y}_{i} & =\sum_{j=1}^{n_{i}} Y_{i j} / n_{i}, \\
S_{i}^{2} & =\sum_{j=1}^{n_{i}}\left(Y_{i j}-\bar{Y}_{i}\right)^{2}, \quad i=1,2 .
\end{aligned}
$$

In this example, we have

$$
\begin{aligned}
n_{1} & =13, n_{2}=11, r_{1}=s_{1}=r_{2}=s_{2}=0 \\
\bar{y}_{1} & =\frac{1}{13} \sum_{j=1}^{13} y_{1 j} \\
& =142.385 \\
\bar{y}_{2} & =\frac{1}{11} \sum_{j=1}^{11} y_{2 j} \\
& =152.545 \\
S_{1}^{2} & =\sum_{j=1}^{13}\left(y_{1 j}-\bar{y}_{1}\right)^{2} \\
& =244097.0769 \\
S_{2}^{2} & =\sum_{j=1}^{11}\left(y_{2 j}-\bar{y}_{2}\right)^{2} \\
& =96840.72727 .
\end{aligned}
$$


Hence we calculate the two-sample $V^{*}$-statistic as follows

$$
\begin{aligned}
V^{*}\left(n_{1}, n_{2}\right) & =\frac{\left\{n_{1}\left(\bar{Y}_{1}-Y_{11}\right)+n_{2}\left(\bar{Y}_{2}-Y_{21}\right)\right\}^{2}}{\left(n_{1}+n_{2}-2\right)\left\{\left(n_{1}+n_{2}-1\right)\left[S_{1}^{2}+S_{2}^{2}\right]-\left[n_{1}\left(\bar{Y}_{1}-Y_{11}\right)+n_{2}\left(\bar{Y}_{2}-Y_{21}\right)\right]^{2}\right\}} \\
& =0.05649 .
\end{aligned}
$$

From Table 1 of percentage points (also given in the Appendix) of the W-exponential statistic (Shapiro and Wilk, 1972), it is seen that this value is greater than the lower $5 \%$ critical value 0.0266 . That is, there is no evidence of non-exponentiality and unequal failure rates of the two failure distributions. Note that we reached the same conclusion as we did in Chapter 3 using the V-exponential statistic.

\subsection{Power Study}

Table 6.1 gives the simulated power of the Shapiro-Wilk test based on $W_{E}(n)$ for $n=20$ and the power of $V^{*}\left(n_{1}, n_{2}\right)$ with $n_{1}=11$ and $n_{2}=10$ for various alternatives. The reason for taking $n_{1}=11$ and $n_{2}=10$ is that $V^{*}\left(n_{1}, n_{2}\right)$ has the same null distribution as the null distribution of the Shapiro-Wilk statistic with $n=n_{1}+n_{2}-$ $1=20$, that is $W_{E}(20)$. The displayed results for $W_{E}(20)$ have been taken from Stephens (1978). From the table, it appears that the power of $V^{*}$ with $n_{1}=11$ and $n_{2}=10$ is comparable to that of the one-sample Shapiro-Wilk statistic with $n=20$ for various alternatives.

In Table 6.2 we compare the power of the one-sample $W$-exponential test for $n=29$ 
Chapter 6. Two-sample $V^{*}$-statistic for Exponentiality for Complete Samples

to the power of the two-sample $V$-exponential and $V^{*}$-exponential tests for $n_{1}=15$, $n_{2}=15$ at the $\alpha=10 \%$ significance level.

We see that the results are close to each other. That is, the three tests ( $W$ exponential, $V$-exponential and $V^{*}$-exponential statistics) seem to be comparable in terms of sensitivity. 
Table 6.1: Estimated Powers of $V^{*}(11,10)$ and $W_{E}(20)$ at $5 \%$ significance level.

\begin{tabular}{|c|c|c|}
\hline & $W_{E}(20)$ & $V^{*}(11,10)$ \\
\hline Gamma (4) & 0.40736 & 0.38822 \\
$\chi^{2}(1)$ & 0.27 & 0.25272 \\
$\chi^{2}(4)$ & 0.21 & 0.16754 \\
$\chi^{2}(6)$ & 0.38 & 0.29486 \\
Inverse- $\chi^{2}(6)$ & 0.1731 & 0.1698 \\
Beta(1,3) & 0.09 & 0.149 \\
Lognormal(0,0.3) & 0.60998 & 0.47034 \\
Lognormal(0,0.7) & 0.12518 & 0.10534 \\
Lognormal(0,1) & 0.21 & 0.20858 \\
Weibull(2.0,1) & 0.72 & 0.52654 \\
Weibull(0.5,1) & 0.63 & 0.698 \\
Half-normal & 0.21 & 0.18752 \\
Half-Cauchy & 0.69 & 0.67892 \\
Uniform(0,1) & 0.76 & 0.72608 \\
Normal & 0.92154 & 0.81778 \\
Power Function(1/5) & 0.57362 & 0.50092 \\
Power Function(1/3) & 0.08194 & 0.05882 \\
Power Function(1/2) & 0.05716 & 0.07686 \\
\hline
\end{tabular}


Table 6.2: Estimated Powers of $V$ and $V^{*}$ exponential tests for $n_{1}=15, n_{2}=15$, and $W$ test for $n=29$, at $10 \%$ significance level.

\begin{tabular}{|c|c|c|c|}
\hline & $W_{E}(29)$ & $V(15,15)$ & $V^{*}(15,15)$ \\
\hline$\chi^{2}(1)$ & 0.5293 & 0.5024 & 0.49122 \\
$\chi^{2}(4)$ & 0.45102 & 0.3672 & 0.38332 \\
$\chi^{2}(6)$ & 0.70026 & 0.5992 & 0.60966 \\
Beta(1,3) & 0.36124 & 0.348 & 0.36228 \\
Lognormal(0,0.3) & 0.87658 & 0.8032 & 0.7935 \\
Lognormal(0,0.7) & 0.2405 & 0.1962 & 0.20392 \\
Lognormal(0,1) & 0.3395 & 0.3474 & 0.35118 \\
Weibull(2.0,1) & 0.92202 & 0.8688 & 0.87008 \\
Weibull(0.5,1) & 0.92768 & 0.9146 & 0.9154 \\
Half-normal & 0.44756 & 0.4144 & 0.42774 \\
Half-Cauchy & 0.85076 & 0.862 & 0.8575 \\
Uniform(0,1) & 0.96218 & 0.9568 & 0.96002 \\
Normal & 0.99594 & 0.9896 & 0.98492 \\
t-distribution(2) & 0.93734 & 0.8988 & 0.80946 \\
t-distribution(3) & 0.96424 & 0.9392 & 0.90004 \\
t-distribution(4) & 0.97706 & 0.9562 & 0.93642 \\
t-distribution(6) & 0.9881 & 0.9764 & 0.9629 \\
Power Function(1/5) & 0.83426 & 0.7764 & 0.7946 \\
Power Function(1/3) & 0.1745 & 0.131 & 0.13612 \\
Power Function(1/2) & 0.1231 & 0.1504 & 0.1602 \\
\hline
\end{tabular}




\section{Chapter 7}

\section{Conclusion}

In this thesis, using the principles of the $\mathrm{W}$-statistic for exponentiality of a single distribution (Shapiro and Wilk, 1972; Samanta and Schwarz, 1988) we proposed the V-exponential statistic for testing exponentiality of two distributions for both complete and censored samples. The proposed statistic turns out to be a normalized ratio of the square of the generalized least squares estimate (also the minimum variance unbiased estimate, that is MVUE) of the common scale parameter to a pooled sum of squares about the samples means. The V-exponential statistic is origin and scale invariant. We proved some important results relating to our proposed twosample V-exponential statistic for testing exponentiality. It has a null distribution that depends only on the sample sizes $n_{1}$ and $n_{2}$. The V-exponential statistic has been presented as two-tailed in the sense that for an unspecified alternative to the exponential, the statistic may shift to either smaller or larger values. We provided some empirical power results for various types of probability distributions under the alternative hypothesis. From these results, it is clear that it has comparative sensitive results for various alternatives. Following the approach of Samanta and Schwarz (1988) the V-exponential statistic was also modified for one or both samples being censored. The modified test statistic has the same null distribution as in the

uncensored case, with a corresponding reduction in sample size(s). In each case, we considered numerical examples to illustrate the applications of the proposed test. 
Further we used Stephens' (1978) approach and proposed a second test statistic called $V^{*}$-exponential statistic for testing forexponentiality of two distributions in the context of complete samples. This statistic has the same null distribution as the W-exponential statistic of Shapiro and Wilk (1972) corresponding to an appropriate sample size.

We also compared the power of the one-sample $W$-exponential test for $n=29$, the two-sample $V$-exponential and $V^{*}$-exponential test for $n_{1}=15, n_{2}=15$ at the $\alpha=10 \%$ significance level. We found that the results are close to each other, that is, the three tests ( $W$-exponential, $V$-exponential and $V^{*}$-exponential statistics) are comparable in terms of sensitivity.

We are not aware of any literature in which exponentiality of two distributions having the same scale parameter has been examined. In the absence of another test, the $V$ and $V^{*}$ exponential tests are useful additions to the current literature on testing exponentiality of two distributions. 


\section{Appendix}

Percentage Points of W-Exponential (Shapiro and Wilk, 1972, page 361)

\begin{tabular}{|c|cccccccc|}
\hline$n$ & 0.005 & 0.01 & 0.025 & 0.05 & 0.95 & 0.975 & 0.99 & 0.995 \\
\hline 3 & 0.2519 & 0.2538 & 0.2596 & 0.2697 & 0.9926 & 0.9981 & 0.9997 & 0.99993 \\
4 & 0.1241 & 0.1302 & 0.1434 & 0.1604 & 0.8581 & 0.9236 & 0.9680 & 0.9837 \\
5 & 0.0845 & 0.0905 & 0.1048 & 0.1187 & 0.6682 & 0.7590 & 0.8600 & 0.9192 \\
6 & 0.0610 & 0.0665 & 0.0802 & 0.0956 & 0.5089 & 0.5842 & 0.6775 & 0.7501 \\
7 & 0.0514 & 0.0591 & 0.0700 & 0.0810 & 0.4162 & 0.4852 & 0.5706 & 0.6426 \\
8 & 0.0454 & 0.0512 & 0.0614 & 0.0710 & 0.3497 & 0.4033 & 0.4848 & 0.5428 \\
9 & 0.0404 & 0.0422 & 0.0537 & 0.0633 & 0.3005 & 0.3454 & 0.4015 & 0.4433 \\
10 & 0.0369 & 0.0404 & 0.0487 & 0.0568 & 0.2525 & 0.2879 & 0.3391 & 0.3701 \\
11 & 0.0339 & 0.0380 & 0.0447 & 0.0528 & 0.2265 & 0.2619 & 0.3039 & 0.3314 \\
12 & 0.0311 & 0.0358 & 0.0410 & 0.0494 & 0.2019 & 0.2364 & 0.2716 & 0.2978 \\
13 & 0.0287 & 0.0337 & 0.0382 & 0.0460 & 0.1829 & 0.2113 & 0.2422 & 0.2642 \\
14 & 0.0265 & 0.0317 & 0.0362 & 0.0428 & 0.1647 & 0.1862 & 0.2131 & 0.2315 \\
15 & 0.0247 & 0.0298 & 0.0334 & 0.0398 & 0.1485 & 0.1669 & 0.1926 & 0.2123 \\
16 & 0.0233 & 0.0280 & 0.0326 & 0.0374 & 0.1355 & 0.1542 & 0.1770 & 0.1931 \\
17 & 0.0222 & 0.0264 & 0.0310 & 0.0352 & 0.1257 & 0.1423 & 0.1614 & 0.1794 \\
18 & 0.0212 & 0.0250 & 0.0294 & 0.0332 & 0.1164 & 0.1311 & 0.1483 & 0.1668 \\
19 & 0.0203 & 0.0238 & 0.0278 & 0.0314 & 0.1071 & 0.1199 & 0.1374 & 0.1452 \\
20 & 0.0196 & 0.0227 & 0.0264 & 0.0302 & 0.1002 & 0.1121 & 0.1286 & 0.1369 \\
21 & 0.0190 & 0.0217 & 0.0250 & 0.0290 & 0.0948 & 0.1054 & 0.1198 & 0.1288 \\
\hline & 0.0185 & 0.0208 & 0.0238 & 0.0278 & 0.0894 & 0.0988 & 0.1118 & 0.1213 \\
\hline
\end{tabular}




\begin{tabular}{|c|cccccccc|}
\hline$n$ & 0.005 & 0.01 & 0.025 & 0.05 & 0.95 & 0.975 & 0.99 & 0.995 \\
\hline 23 & 0.0181 & 0.0201 & 0.0230 & 0.0266 & 0.0836 & 0.0933 & 0.1043 & 0.1142 \\
24 & 0.0177 & 0.0194 & 0.0224 & 0.0256 & 0.0788 & 0.0882 & 0.0984 & 0.1071 \\
25 & 0.0173 & 0.0188 & 0.0218 & 0.0248 & 0.0749 & 0.0836 & 0.0927 & 0.1000 \\
26 & 0.0169 & 0.0182 & 0.0213 & 0.0240 & 0.0712 & 0.0791 & 0.0885 & 0.0948 \\
27 & 0.0165 & 0.0177 & 0.0208 & 0.0232 & 0.0687 & 0.0747 & 0.0843 & 0.0896 \\
28 & 0.0161 & 0.0172 & 0.0203 & 0.0225 & 0.0649 & 0.0706 & 0.0801 & 0.0859 \\
29 & 0.0157 & 0.0168 & 0.0198 & 0.0219 & 0.0621 & 0.0671 & 0.0759 & 0.0822 \\
30 & 0.0153 & 0.0164 & 0.0193 & 0.0213 & 0.0593 & 0.0643 & 0.0719 & 0.0786 \\
\hline
\end{tabular}




\section{Bibliography}

[1] Aitken, A.C. (1935). On least squares and linear combination of observations. Proc. Roy. Soc. Edin., 55, 42-48.

[2] Anderson, T.W. and Darling, D.A. (1952). Asymeptotic theory of certain "goodness of fit" criteria based on stochastic process. Am. Math. Statist., 23, 193-212.

[3] Angus, J.E. (1989). A note on the use of the Shpiro-Wilk test of exponentiality for complete samples. Communications in Statistics - Theory and Methods, 18, $1819-1830$.

[4] Bain, L.J. (1978). Statistical Analysis of reliability and life-testing models - Theory and Methods, Marcel Dekker, New York.

[5] Bartholomew, D.J. (1957). Testing for departure from the exponential distribution. Biometrika, 44, 253-257.

[6] Basu, D. (1955). On statistics independent of a complete sufficient statistic. Sankhya, 15, 377-380.

[7] Brain, C.W. and Shapiro, S.S. (1983). A Regression test for exponentiality: Censored and complete samples. Technometrics, 25, 69-76.

[8] Cox, D.R. (1964). Some applications of exponential ordered scores. J. R. Statist. Soc. , B 26, 103-110.

[9] Cox, D.R. and Lewis, P.A.W. (1966). Statistical Analysis of Series of Events., London: Methuen. 
[10] Currie, I.D. (1980). The upper tail of the distribution of W-exponential. Scandinavian Journal of Statistics, 7, 147-149.

[11] Darling, D.A. (1953). On a class of problems related to the random division of an interval. Am. Math. Statist., 24, 239-253.

[12] Davis, C.S. and Stephens, M.A. (1977). The covariance matrix of normal order statistics. Commun. Statist .-Simula. Computa. B6, 135-149.

[13] Epstein, B. (1960). Tests for the validity of the assumption that the underlying distributions of life is exponential. (Part I and II.) Technometrics 2, 83-101, $167-183$.

[14] Epstein, B. and Sobel, M. (1954). Some theorems relevant to life testing from an exponential distribution. The Annals of Mathematical Statistics, 25, 373-381.

[15] Fish, R.A. (1950). Statistical Methods for Research Workers. 11th Edit., Hafner Publishing Company, NewYork.

[16] Hogg, R.V. and Craig, A.J. (1956). sufficient statistics in elementary distribution theory. Sankhya, 17, 209-216.

[17] Jackson, O.A.Y. (1967). An analysis of departures from the exponential distribution.. J. R. Statist. Soc., B 29, 540-549.

[18] Kendall, M.G. and Stuart, A. (1961). The advance Theory of Statistics, 2. London, C.Griffin and Co.

[19] Kolmogorov, A.N. (1933). Sulla determinazione empirica di una legge di distibuziane. Giorna. Ist. Attuari. , 4, 83-91. 
[20] Lehmann, E.L. (1959). Testing Statistical Hypothesis. John Wiley and Sons, Inc., New York.

[21] Lloyd, E.H. (1952). Least squares estimation of location and scale parameters using order statistics. Biometrika, 39, 88-95.

[22] Proschan, F. (1963). Theoretical explanation of observed decreasing failure rate. Technometrics, 5, 375-384.

[23] Rao, C.R. (1973). Linear Statistical Inference and Its Applications (2nd Edition) Wiley, New York.

[24] Samanta, M. (1985). On a geometrical derivation of the null distribution of W-exponential. Communications in Statistics - Theory and Methods, 14, 29472950.

[25] Samanta, M. and Schwarz, C.J. (1987). On the Shapiro-Wilk test for exponentiality based on censored data. Technical report 181, Department of Statistics, University of Manitoba.

[26] Samanta, M. and Schwarz, C.J. (1988). The Shapiro-Wilk test for exponentiality based on censored data. Journal of the American Statistical Association, 83, $528-531$.

[27] Shapiro, S.S. and Wilk, M.B. (1965). An analysis of variance test for normality ( complete samples ). Biometrika, 52, 591-611.

[28] Shapiro, S.S. and Wilk, M.B. (1968). Testing for normality with specified parameters. Unpublished paper. 
[29] Shapiro, S.S. and Wilk, M.B. (1972). An analysis of variance test for exponential distribution ( complete samples ). Technometrics, 14, 355-370.

[30] Shapiro, S.S., Wilk, M.B. and Chen, H.J. (1968). A comparative study of various tests for normality. J. Amer. Stat. Assoc. , 63, 1343-1372.

[31] Stephens, M.A. (1974). EDF statistics for goodness of fit and some comparisons. J.Amer.Statist. Assoc., 69, 730-737.

[32] Stephens, M.A. (1975). Asymptotic properties for covariance matrices of order statistics. Biometrika, 62, 23-28.

[33] Stephens, M.A. (1976). Extensions to the W-test for normality. Technical report, Department of Statistics, Stanford University.

[34] Stephens, M.A. (1978). On the $\mathrm{W}$ test for exponentiality with origin known. Technometrics, 20, 33-35.

[35] Stephens, M.A. (1986). Goodness of fit for censored data. Technical report, Department of Statistics, Stanford University.

[36] Wilk, M.B. and Shapiro, S.S. (1968). The joint assement of normality of several independent samples. Technometrics, 10, 825-839. 